\title{
REAL SUBMANIFOLDS OF CODIMENSION TWO IN COMPLEX MANIFOLDS ${ }^{1}$
}

BY

\author{
HON-FEI LAI
}

\begin{abstract}
The equivalence problem for a real submanifold $M$ of dimension at least eight and codimension two in a complex manifold is solved under a certain nondegeneracy condition on the Levi form. If the Levi forms at all points of $M$ are equivalent, a normalized Cartan connection can be defined on a certain principal bundle over $\boldsymbol{M}$. The group of this bundle can be described by means of the osculating quartic of $M$ or the prolongation of the graded Lie algebra of type $\mathrm{g}_{-2} \oplus \mathrm{g}_{-1}$ defined by the Levi form.
\end{abstract}

1. Introduction. Pseudoconformal geometry is concerned with the invariants of a real submanifold $M$ of a complex manifold under biholomorphic transformations of the ambient manifold. Local invariants for hypersurfaces have been studied by E. Cartan in the case of $\mathbf{C}^{2}$, and recently by Tanaka and Chern and Moser in the general case (see [1] for references). Since then, a lot of work has been done by various authors. For higher codimensions, however, the only results known are those of Tanaka [7] on the Lie algebra of infinitesimal automorphisms of the submanifold.

There are two essential distinctions between codimension one and higher codimensions. Firstly, a hypersurface is always RC-regular, whereas in higher codimensions the RC-singular points determine the characteristic classes of $M$ [5]. Secondly, the Levi form of a hypersurface, being a Hermitian form, is completely determined by the number of positive and negative eigenvalues, and if the Levi forms at all points are nondegenerate, then they are all equivalent (assuming connectedness); in codimension $p>1$, the Levi form is a Hermitian mapping into a $p$-dimensional Euclidean space, and there are many more invariants, and even if the Levi forms at all points are nondegenerate in any reasonable sense, they need not be equivalent.

In $\$ 2$ of this paper we study the Levi form for codimension two, and introduce a reasonable nondegeneracy condition that will ensure that the Lie algebra of infinitesimal automorphisms of $M$ is finite dimensional. The general scheme [7], [8] of prolongation of a generalized graded Lie algebra, which we will just refer to as a graded Lie algebra, is then used in $\$ 3$ to compute the Lie algebra (for $\operatorname{dim} M \geqslant 8$ ) which governs the pseudoconformal structure studied in the following three sections. Unexpectedly, this Lie algebra turns out to be of very low dimension

Received by the editors March 13, 1979.

AMS (MOS) subject classifications (1970). Primary 53B15, 17B99; Secondary 53B35.

Key words and phrases. Pseudoconformal invariants, Levi form, prolongation of graded Lie algebras, Cartan connection.

'This research was partially supported by NSF Grant No. MCS 77-01592. 
compared with the case of a hypersurface. The same Lie algebra is identified with the Lie algebra of the group of projective automorphisms of a standard quartic in $\S 7$. Lastly, in $\S 8$ we construct a Cartan connection on a suitable bundle over $M$, from which all local pseudoconformal invariants of $M$ can be derived by successive covariant differentiations of the torsion and curvature. Throughout this paper, only local properties are studied. However, the fact that we are dealing with evendimensional manifolds brings a good hope of global, particularly topological properties.

2. The Levi form for codimension 2. Let $M$ be a $(2 n+p)$-dimensional real submanifold of an $(n+p)$-dimensional complex manifold $N$. We want to find all the local pseudoconformal invariants of $M$. There is no loss of generality in assuming that $N=\mathrm{C}^{n+p}$ with the usual almost complex structure $J$. We assume that $M$ is RC-regular [5], that is, the maximal complex subspace of the tangent space of $M$ at each point is $n$-dimensional. In this case there is a well-known pseudoconformal invariant, the Levi form [3]

$$
\tilde{L}: H(M) \times H(M) \rightarrow T(N) / T(M),
$$

where $T(M)$ is the tangent space of $M$, and $H(M)=T(M) \cap J(T(M))$ is the maximal complex subspace of $T(M)$; it is defined by

$$
\tilde{L}(X, Y)=\tilde{\pi} J[J X, Y]
$$

for all vector fields $X, Y$ on $M$ belonging to $H(M)$, where $\tilde{\pi}$ is the projection of $T(N)$ onto $T(N) / T(M)$.

For our purpose, it is more convenient to think of the Levi form as a bilinear map (over functions)

$$
L: H(M) \times H(M) \rightarrow T(M) / H(M)
$$

defined by

$$
L(X, Y)=\pi[X, J Y]
$$

for all vector fields $X, Y$ on $M$ belonging to $H(M)$, where $\pi$ is the projection of $T(M)$ onto $T(M) / H(M)$. We have

$$
L(X, Y)=L(Y, X)=L(J X, J Y)
$$

for all $X, Y \in H(M)$.

We now restrict to the case $p=2$ and study the matrix representation of $L$. Consider, in more general terms, a Hermitian bilinear map

$$
L: V \times V \rightarrow W
$$

where $V$ is a $2 n$-dimensional real vector space with almost complex structure $J$, and $W$ is a two-dimensional real vector space. Let $\left\{e_{1}, \ldots, e_{n}, J e_{1}, \ldots, J e_{n}\right\}$ be a basis of $V$, and $\left\{e_{1^{\prime}}, e_{2^{\prime}}\right\}$ a basis of $W$. Then the Hermitian map $L$ can be written $L(X, Y)=L^{1^{\prime}}(X, Y) e_{1^{\prime}}+L^{2^{\prime}}(X, Y) e_{2^{\prime}}$, where $L^{1^{\prime}}, L^{2^{\prime}}$ are Hermitian forms on $V$. Complexify and get a Hermitian sesquilinear map

$$
L: V \times V \rightarrow W \otimes \mathbf{C},
$$

that is, $L\left(z X, z^{\prime} Y\right)=z \bar{z}^{\prime} L(X, Y)$ for all $X, Y \in V, z, z^{\prime} \in \mathbf{C}$, when $V$ is regarded as a complex vector space by means of $J$. The matrices of the complexified forms 
$L^{(\alpha)}: V \times V \rightarrow \mathrm{C}$ are $2 g^{\alpha}=\left(2 g_{i j}^{\alpha}\right)_{i, j}$ for $\alpha=1^{\prime}, 2^{\prime}$, where

$$
2 g_{i j}^{\alpha}=L^{\alpha}\left(e_{i}, e_{j}\right)+\sqrt{-1} L^{\alpha}\left(e_{i}, J e_{j}\right) .
$$

We need to find the invariants of $L$ under the action of $\operatorname{GL}(n, C)$ on $V$ and $\mathrm{GL}(2, \mathbf{R})$ on $W$.

Definition 2.1. A Hermitian sesquilinear map $L: V \times V \rightarrow W \otimes \mathbf{C}$ with $\operatorname{dim}_{\mathrm{C}} V$ $=n \geqslant 2$ is said to be most nondegenerate if, for some bases $\left\{e_{1}, \ldots, e_{n}\right\}$ of $V$ over $\mathbf{C}$ and $\left\{e_{1^{\prime}}, e_{2^{\prime}}\right\}$ of $W, \operatorname{det}\left(\lambda g^{1^{\prime}}-\mu g^{2^{\prime}}\right)$ is not identically zero and factors into $n$ nonproportional linear factors in $\lambda, \mu$. A symmetric bilinear map $L: V \times V \rightarrow W$ satisfying $L(J X, J Y)=L(X, Y)$ for all $X, Y \in V$ is said to be most nondegenerate if its complexification is.

It is easily seen that this definition is actually independent of the choice of bases in $V$ and $W$. Moreover, under a change of bases in $W$, we have $g^{* \alpha}=b_{\beta}^{\alpha} g^{\beta}$ for $\left(b_{\beta}^{\alpha}\right) \in \mathrm{GL}(2, \mathbf{R})$. Since there is only a finite number of ratios $\lambda$ : $\mu$ such that $\operatorname{det}\left(\lambda g^{1^{\prime}}-\mu g^{2^{\prime}}\right)=0$, it follows that, for an open dense set in $\operatorname{GL}(2, \mathbf{R})$, the matrices $g^{1^{\prime}}, g^{2^{\prime}}$ are both nonsingular.

LEMMA 2.1. Let $g^{1^{\prime}}, g^{2^{\prime}}$ be nonsingular Hermitian matrices of order $n$ such that $\operatorname{det}\left(\lambda g^{1^{\prime}}-g^{2^{\prime}}\right)$ factors into $n$ nonproportional linear factors in $\lambda$. The roots occur in conjugate pairs. Denote them by $\lambda_{1}, \bar{\lambda}_{1}, \lambda_{3}, \bar{\lambda}_{3}, \ldots, \lambda_{2 m-1}, \bar{\lambda}_{2 m-1}, \lambda_{2 m+1}, \ldots, \lambda_{n}$, where $0 \leqslant m \leqslant n / 2, \lambda_{1}, \ldots, \lambda_{2 m-1}$ are not real, and $\lambda_{2 m+1}, \ldots, \lambda_{n}$ are real. Then there is a basis $\left\{e_{1}, \ldots, e_{n}\right\}$ of $V$ such that:

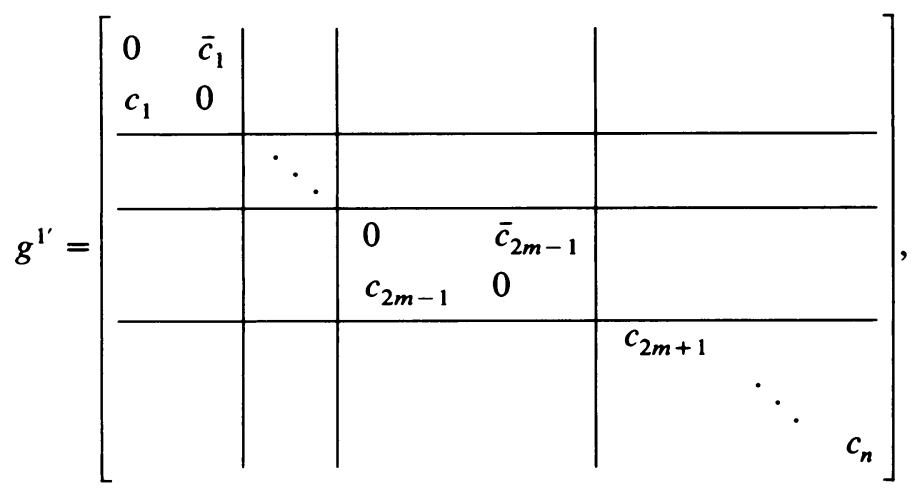

$g^{2^{\prime}}=\left[\begin{array}{ll|l|l|l|l}0 & \bar{\lambda}_{1} \bar{c}_{1} & & & & \\ \lambda_{1} c_{1} & 0 & & & & \\ \hline & & \ddots & & & \\ \hline & & 0 & \bar{\lambda}_{2 m-1} \bar{c}_{2 m-1} & & \\ \hline & & \lambda_{2 m-1} c_{2 m-1} & 0 & & \\ \hline & & & & \lambda_{2 m+1} c_{2 m+1} & \ddots \\ & & & & & \lambda_{n} c_{n}\end{array}\right]$.


Moreover, $c_{1}, \ldots, c_{2 m-1}$ can be chosen to be 1 , and $c_{2 m+1}, \ldots, c_{n}$ to be \pm 1 . When the order $\lambda_{1}, \ldots, \lambda_{n}$ is fixed, the basis $\left\{e_{1}, \ldots, e_{n}\right\}$ giving the above canonical forms for $g^{1^{\prime}}, g^{2^{\prime}}$ is determined up to transformations of the group

$$
\begin{aligned}
\left\{\operatorname{diag}\left(e^{a_{1}}, e^{-\bar{a}_{1}}, \ldots, e^{a_{2 m-1}}, e^{-\bar{a}_{2 m-1}}, e^{i b_{2 m+1}}, \ldots, e^{i b_{n}}\right):\right. & \\
& \left.a_{1}, a_{3}, \ldots, a_{2 m-1} \in \mathbf{C}, b_{2 m+1}, \ldots, b_{n} \in \mathbf{R}\right\},
\end{aligned}
$$

where $i=\sqrt{-1}$.

Proof. The zeros of $\operatorname{det}\left(\lambda g^{1^{\prime}}-g^{2^{\prime}}\right)$ occur in conjugate pairs since

$$
\operatorname{det}\left(\lambda g^{1^{\prime}}-g^{2^{\prime}}\right)=\operatorname{det}\left(\lambda^{t} g^{1^{\prime}}-{ }^{t} g^{2^{\prime}}\right)=\overline{\operatorname{det}\left(\bar{\lambda} g^{1^{\prime}}-g^{2^{\prime}}\right)} \text {. }
$$

Note that

$$
\operatorname{det}\left(\lambda g^{1^{\prime}}-g^{2^{\prime}}\right)=\operatorname{det}\left(g^{1^{\prime}}\right) \cdot \operatorname{det}\left(\lambda I-\left(g^{1^{\prime}}\right)^{-1} g^{2^{\prime}}\right),
$$

so $\lambda_{i}$ are the eigenvalues of $\theta=\left(g^{1^{\prime}}\right)^{-1} g^{2^{\prime}}$. Under a change of basis in $V$,

$$
g^{* \alpha}={ }^{t} A g^{\alpha} \bar{A} \text { for } \alpha=1^{\prime}, 2^{\prime},
$$

so

$$
\left(g^{* 1^{\prime}}\right)^{-1} g^{* 2^{\prime}}=\bar{A}^{-1}\left(g^{1^{\prime}}\right)^{-1} g^{2^{\prime}} \bar{A} .
$$

Thus the matrix of $\theta$ changes by similarity. Since the eigenvalues $\lambda_{i}$ are distinct, we can find a basis $\left\{e_{1}, \ldots, e_{n}\right\}$ of $V$ for which $\theta$ is diagonal, $\theta=\operatorname{diag}\left(\lambda_{1}, \ldots, \lambda_{n}\right)$, where $\lambda_{2 i}=\bar{\lambda}_{2 i-1}$ for $1 \leqslant i \leqslant m$, and $\lambda_{2 m+1}, \ldots, \lambda_{n}$ are real. Then $g^{2^{\prime}}=g^{1^{\prime} \theta}$, or $g_{i j}^{2^{\prime}}=g_{i j}^{1^{\prime}} \lambda_{j}$. The Hermitian property of $g^{\alpha}$ gives $g_{i j}^{1^{\prime}} \lambda_{j}=\overline{g_{j i}^{1^{\prime}} \lambda_{i}}$, or $g_{i j}^{1^{\prime}}\left(\lambda_{j}-\bar{\lambda}_{i}\right)=0$. Therefore $g_{i j}^{1^{\prime}}=0$ unless $\{i, j\}=\{2 k, 2 k-1\}$ with $1 \leqslant k \leqslant m$ or else $i=j \geqslant 2 m$ +1 . Consequently $g^{\alpha}$ are of the form stated in the lemma. Moreover, by taking suitable multiples of $e_{1}, \ldots, e_{n}$, we can make $c_{1}=c_{3}=\ldots=c_{2 m-1}=1$ and $c_{2 m+1}, \ldots, c_{n}= \pm 1$. The rest is clear.

At this point it is convenient to set a convention. If the roots $\operatorname{of} \operatorname{det}\left(\lambda g^{1^{\prime}}-g^{2^{\prime}}\right)$ are $\lambda_{1}, \ldots, \lambda_{n}$ with $\lambda_{2 i}=\bar{\lambda}_{2 i-1} \notin \mathbf{R}$ for $1 \leqslant i \leqslant m$ and $\lambda_{2 m+1}, \ldots, \lambda_{n} \in \mathbf{R}$, we write

$$
\left\{\begin{array}{l}
\tilde{2 i=2 i}-1 \text { for } 1 \leqslant i \leqslant m, \\
2 i-1=2 i \text { for } 1 \leqslant i<m, \\
\tilde{j}=j \text { for } 2 m+1 \leqslant j \leqslant n .
\end{array}\right.
$$

Then we can write $\lambda_{i}=\bar{\lambda}_{i}$ for $1 \leqslant i \leqslant n$.

Lemma 2.2. Let $\operatorname{dim}_{\mathbf{C}} V=n \geqslant 3$. Then among the roots $\lambda_{1}, \ldots, \lambda_{n}$ in Lemma 2.1, any three real parameters can be made equal to three different but otherwise arbitrary real numbers by suitable choice of basis in $W$. After this normalization, two Hermitian maps $L, L^{\prime}: V \times V \rightarrow \mathrm{C}^{2}$ are equivalent under the actions of $\mathrm{GL}(n, \mathrm{C})$ on $V$ and $\mathrm{GL}(2, \mathbf{R})$ on $\mathrm{C}^{2}$ (that is, there exist $A \in \mathrm{GL}(n, \mathbf{C})$ and $B \in \mathrm{GL}(2, \mathbf{R})$ such that $L^{\prime}(A v, A w)=B(L(v, w))$ for all $\left.v, w \in V\right)$ if and only if $\left\{\lambda_{1}, \ldots, \lambda_{n}\right\}=$ $\left\{\lambda_{1}^{\prime}, \ldots, \lambda_{n}^{\prime}\right\}$.

Proof. Under a change of basis in $\mathbf{C}^{2}$ by the action of $\mathrm{GL}(2, \mathbf{R})$, the roots $\lambda_{i}$ are transformed by the same projective transformation with three parameters. The rest follows easily. 
3. The prolongation of the graded Lie algebra. Whereas in the case of a hypersurface there is a hint that the Lie algebra of infinitesimal pseudoconformal transformations of $M$ should be the Lie algebra of the group of projective transformations of the osculating hyperquadric, for codimension 2 there is no obvious analogue. However, according to the scheme of Tanaka [7], the associated Lie algebra is the prolongation of a certain graded Lie algebra of type $g_{-2} \oplus g_{-1}$. In this section we study this graded Lie algebra.

Let $M$ be an RC-regular $(2 n+2)$-dimensional real submanifold of $\mathbf{C}^{n+2}, n>2$, whose Levi forms at all points are equivalent and are most nondegenerate. We can then associate a graded Lie algebra $g_{-2} \oplus g_{-1}$ modeled after the Levi form of $M$, by taking $\mathfrak{g}_{-1}=V$ to be the maximal complex subspace of the tangent space to $M$ at one point $p$ and $\mathfrak{g}_{-2}=W$ to be the quotient $T_{p}(M) / \mathfrak{g}_{-1}$, and by defining $[X, Y]=$ $-L(X, J Y)$ for $X, Y \in g_{-1}$, where $L$ is the Levi form defined in (2.1), and by putting all other brackets to be zero. Thus the new bracket is the ordinary Lie bracket of vector field extensions projected into $g_{-2}$. The assumption of most nondegeneracy implies that $\left[\mathfrak{g}_{-1}, \mathfrak{g}_{-1}\right]=\mathfrak{g}_{-2}$ since $n \geqslant 2$. The graded Lie algebra $\mathfrak{g}_{-2} \oplus \mathfrak{g}_{-1}$ is of the type called pseudo-complex by Tanaka [8, p. 72], and for such a graded Lie algebra Tanaka has given in the same paper a general scheme of prolongation similar to that of [7], and if the prolongation $g$ is finite-dimensional, then the Lie algebra of all infinitesimal pseudoconformal automorphisms of $M$ is of dimension $\leqslant \operatorname{dim} g$. In this section we calculate the prolongation $g$. Its Lie group is used to solve the equivalence problem for $M$ in $\$ 4$ and to define a Cartan connection on a certain bundle over $M$, describing all local pseudoconformal invariants of $M$, in $\S 8$. We will use [,] to denote the bracket operation in the Lie algebra $g_{-1} \oplus g_{-2}$. For the rest of the paper we will let the indices $\alpha, \beta, \gamma$ run from $1^{\prime}$ to $2^{\prime}$ and $i, j, k, l$ from 1 to $n$.

Take a basis $\left\{e_{1}, \ldots, e_{n}\right\}$ of $g_{-1}$ over $\mathrm{C}$ and a basis $\left\{e_{1^{\prime}}, e_{2^{\prime}}\right\}$ of $g_{-2}$. Write $[X, Y]=[X, Y]^{(\beta)} e_{\beta}$ (summation) for all $X, Y \in g_{-1}$, and define the Hermitian matrices $g^{\alpha}=\left(g_{i j}^{\alpha}\right)_{i, j}$ by

$$
2 g_{i j}^{\alpha}=\left[e_{i}, J e_{j}\right]^{(\alpha)}-\sqrt{-1}\left[e_{i}, e_{j}\right]^{(\alpha)}
$$

for each $\alpha, i, j$, as in (2.3). The assumption of most nondegeneracy enables us to choose $e_{1^{\prime}}, e_{2^{\prime}}$ such that $g^{1^{\prime}}, g^{2^{\prime}}$ are both nonsingular. Then $\left(g^{1^{\prime}}\right)^{-1} g^{2^{\prime}}$ has distinct eigenvalues $\lambda_{1}, \ldots, \lambda_{n}$ satisfying $\lambda_{i}=\bar{\lambda}_{i}$, where $\tilde{i}$ is defined in (2.4), for $1<i<n$. We also choose a basis of $V$ such that $g^{1^{\prime}}$ and $g^{2^{\prime}}$ have the normal forms described in Lemma 2.1.

For any $n$-tuple $\left(\zeta^{1}, \ldots, \zeta^{n}\right)$ of complex numbers, we define

$$
\zeta_{j}^{(\alpha)}=g_{j k}^{\alpha} \bar{\zeta}^{k} \quad \text { for each } \alpha, j,
$$

where summation over $k$ is implied. If $\left(\zeta^{1}, \ldots, \zeta^{n}\right)$ and $\left(\zeta^{\prime \prime}, \ldots, \zeta^{\prime n}\right)$ are any two $n$-tuples of complex numbers and $\zeta^{i}=\xi^{i}+\sqrt{-1} \eta^{i}, \zeta^{\prime i}=\xi^{\prime \prime}+\sqrt{-1} \eta^{\prime \prime}$, it is easy to verify that

$$
\zeta_{j}^{(\alpha)} \zeta^{\prime j}-\zeta_{j}^{\prime(\alpha)} \zeta^{j}=\sqrt{-1}\left[\xi^{j} e_{j}+\eta^{j} J e_{j}, \xi^{\prime k} e_{k}+\eta^{\prime k} J e_{k}\right]^{(\alpha)}
$$


for each $\alpha$. Hence

Proposition 3.1. The graded Lie algebra $g_{-2} \oplus g_{-1}$ is isomorphic to the subalgebra of $\mathrm{gl}(n+3, \mathrm{C})$,

$$
\left\{\left[\begin{array}{cc|c|c}
0 & 0 & \zeta_{j}^{\left(1^{\prime}\right)} & \sqrt{-1} x \\
0 & 0 & \zeta_{j}^{\left(2^{\prime}\right)} & \sqrt{-1} y \\
\hline 0 & 0 & 0 & \zeta^{i} \\
\hline 0 & 0 & 0 & 0
\end{array}\right]: x, y \in \mathbf{R}, \zeta^{i} \in \mathbf{C} \text { for } i=1, \ldots, n\right\}
$$

graded naturally by the block matrices, that is, $\mathfrak{g}_{-2}$ is described by $x, y$ and $\mathfrak{g}_{-1}$ by $\zeta^{i}$.

Following the scheme of Tanaka, we let $g_{0}$ be the Lie algebra of all derivations of $g_{-2} \oplus g_{-1}$ preserving the almost complex structure on $g_{-1}$, that is $g_{0}$ is the set of all pairs $(\rho, \sigma)$, where $\rho: \mathfrak{g}_{-2} \rightarrow \mathfrak{g}_{-2}$ and $\sigma: \mathfrak{g}_{-1} \rightarrow \mathfrak{g}_{-1}$ are linear maps over $\mathbf{R}, \mathbf{C}$ respectively, satisfying

$$
\rho([X, Y])=[\sigma(X), Y]+[X, \sigma(Y)] \text { for all } X, Y \in \mathfrak{g}_{-1} .
$$

Proposition 3.2. Under the representation of Proposition 3.1, $\mathrm{g}_{0}$ is represented by

$$
\left\{\left[\begin{array}{ll|l|l}
a & 0 & & \\
0 & a & & \\
\hline & & \frac{1}{2} a I+\tau & \\
\hline & & 0
\end{array}\right]: a \in \mathbf{R}, \tau=\operatorname{diag}\left(\tau^{i}\right), \quad \tau^{i}=-\bar{\tau}^{i} \text { for } i=1, \ldots, n\right\}
$$

provided that $n \geqslant 3$.

Proof. Take a pair $(\rho, \sigma) \in \mathrm{g}_{0}$ and let $\left(\rho_{\beta}^{\alpha}\right),\left(\sigma_{j}^{i}\right)$ be the matrices of $\rho, \sigma$ relative to the bases $\left\{e_{\alpha}\right\}$ and $\left\{e_{i}\right\}$ respectively. Take $X=\zeta^{i} e_{i}, Y=\zeta^{\prime \prime} e_{i}$. Then equations (3.3), (3.4) give

$$
\begin{aligned}
\rho_{\beta}^{\alpha} g_{i j}^{\beta}\left(\zeta^{i} \bar{\zeta}^{\prime j}-\zeta^{\prime i} \bar{\zeta}^{j}\right) & =g_{i j}^{\alpha}\left(\sigma_{k}^{i} \zeta^{k} \bar{\zeta}^{\prime j}-\zeta^{\prime \prime} \bar{\sigma}_{k}^{j} \bar{\zeta}^{k}\right)+g_{i j}^{\alpha}\left(\zeta^{i} \bar{\sigma}_{k}^{j} \bar{\zeta}^{\prime k}-\sigma_{k}^{i} \zeta^{\prime k} \zeta^{j}\right) \\
& =\left(g_{k j}^{\alpha} \sigma_{i}^{k}+g_{i k}^{\alpha} \bar{\sigma}_{j}^{k}\right)\left(\zeta^{i} \bar{\zeta}^{\prime j}-\zeta^{\prime \prime} \bar{\zeta}^{j}\right) .
\end{aligned}
$$

Since $\zeta^{i}, \zeta^{\prime j}$ are arbitrary, we must have

$$
\rho_{\beta}^{\alpha} g_{i j}^{\beta}=g_{k j}^{\alpha} \sigma_{i}^{k}+g_{i k}^{\alpha} \bar{\sigma}_{j}^{k}
$$

or

$$
\rho_{\beta}^{\alpha} g^{\beta}={ }^{t} \sigma g^{\alpha}+g^{\alpha} \bar{\sigma} \quad \text { for } \alpha=1^{\prime}, 2^{\prime} .
$$

Recall that $g^{1^{\prime}}, g^{2^{\prime}}$ are both nonsingular and that $\theta=\left(g^{1^{\prime}}\right)^{-1} g^{2^{\prime}}$ has distinct eigenvalues $\lambda_{i}$. Then $\phi=\left(g^{2^{\prime}}\right)^{-1} g^{1^{\prime}}$ has distinct eigenvalues $\mu_{i}=1 / \lambda_{i}$. Equation (3.5) can now be written

$$
\left\{\begin{array}{l}
\rho_{1^{\prime}}^{1^{\prime}} I+\rho_{2^{\prime}}^{1^{\prime}} \theta=\bar{\sigma}+\left(g^{1^{\prime}}\right)^{-1}\left({ }^{t} \sigma\right) g^{1^{\prime}} \\
\rho_{1^{\prime}}^{2^{\prime} \phi}+\rho_{2^{\prime}}^{2^{\prime}} I=\bar{\sigma}+\left(g^{2^{\prime}}\right)^{-1}\left({ }^{t} \sigma\right) g^{2^{\prime}}
\end{array}\right.
$$


Write $\rho=\left[\begin{array}{ll}a & b \\ c & d\end{array}\right]$ and $\sigma^{\prime}=\left(g^{1^{\prime}}\right)^{-1}\left({ }^{t} \sigma\right) g^{1^{\prime}}=\left(\sigma_{j}^{\prime \prime}\right)$. Then $\left(g^{2^{\prime}}\right)^{-1}\left({ }^{t} \sigma\right) g^{2^{\prime}}=\phi \sigma^{\prime} \theta$, so (3.6) becomes

$$
\left\{\begin{array}{l}
a \delta_{j}^{i}+b \lambda_{i} \delta_{j}^{i}=\bar{\sigma}_{j}^{i}+\sigma_{j}^{\prime i}, \\
c \mu_{i} \delta_{j}^{i}+d \delta_{j}^{i}=\bar{\sigma}_{j}^{i}+\mu_{i} \sigma_{j}^{\prime i} \lambda_{j}=\bar{\sigma}_{j}^{i}+\sigma_{j}^{\prime \prime} \lambda_{j} / \lambda_{i} .
\end{array}\right.
$$

From these two equations, using the nonproportionality of $\lambda_{i}$, we conclude that

$$
\sigma_{j}^{i}=0 \quad \text { whenever } i \neq j
$$

and

$$
a+b \lambda_{i}=\bar{\sigma}_{i}^{i}+\sigma_{i}^{\prime i}=c \mu_{i}+d \text { for all } i .
$$

It follows that $a-d=c / \lambda_{i}-b \lambda_{i}$. Take two different indices $i, j$, and get $c / \lambda_{i}-b \lambda_{i}=c / \lambda_{j}-b \lambda_{j}$, or $c=-\lambda_{i} \lambda_{j} b$. It follows that

$$
b=c=0 \text { provided } n \geqslant 3 \text {. }
$$

So, assuming that $n \geqslant 3$, we have, from (3.9),

$$
a=d=\bar{\sigma}_{i}^{i}+\sigma_{i}^{i} \text { for all } i .
$$

Write $\sigma=\frac{1}{2} a I+\tau$. Then (3.8) and (3.11) imply that $\tau$ is a diagonal matrix $\operatorname{diag}\left(\tau^{1}, \ldots, \tau^{n}\right)$ satisfying

$$
g^{1^{\prime}} \bar{\tau}+\tau g^{1^{\prime}}=0
$$

(which is actually equivalent to $g^{2^{\prime}} \bar{\tau}+\tau g^{2^{\prime}}=0$ ).

Since $g^{1^{\prime}}$ is in the normal form described in Lemma 2.1, we have $g_{i j}^{1^{\prime}} \neq 0$ if and only if $j=\tilde{i}$. Therefore (3.12), which can be written as $g_{i j}^{1^{\prime}}\left(\bar{\tau}^{j}+\tau^{i}\right)=0$ for all $i, j$, yields the relations

$$
\tau^{i}=-\bar{\tau}^{i} \text { for } i=1, \ldots, n .
$$

(In particular, if $g^{1^{\prime}}$ is diagonal, then $\tau$ is a purely imaginary diagonal matrix.)

REMARK. Here $g_{0}$ is really represented in the affine algebra aff $(n+2, C)$. It can also be represented in $\operatorname{sl}(n+3, \mathrm{C})$ by subtracting

$$
\frac{1}{n+3}\left\{\frac{n+4}{2} a+\operatorname{tr} \tau\right\} I
$$

from the matrix in Proposition 3.2, thereby getting

$$
\left[\begin{array}{cc|c|c}
-(n+2) a^{\prime}-\frac{1}{3} \operatorname{tr} \tau^{\prime} & 0 & & \\
0 & -(n+2) a^{\prime}-\frac{1}{3} \operatorname{tr} \tau^{\prime} & & \\
\hline & a^{\prime} I+\tau^{\prime} & \\
\hline & & (n+4) a^{\prime}-\frac{1}{3} \operatorname{tr} \tau^{\prime}
\end{array}\right]
$$

where $a^{\prime}=-a / 2(n+3)$ and $\tau^{\prime}=\tau-(\operatorname{tr} \tau /(n+3)) I$, so $\tau^{\prime}$ also satisfies (3.13).

In the case $n=2$, on the other hand, (3.9) only yields $c=-\lambda_{1} \lambda_{2} b$ instead of $b=c=0$. It turns out that in this case the Lie algebra $g_{0}$ is 4-dimensional. The 
calculations and results are more complicated than in the case $n>3$, and we do not show them here.

Proposition 3.3. $\mathrm{g}_{1}=0$, provided $n \geqslant 3$.

Proof. $g_{1}$ consists of all pairs $\left(\sigma_{-2}, \sigma_{-1}\right)$, where $\sigma_{-2}: \mathfrak{g}_{-2} \rightarrow \mathfrak{g}_{-1}$ and $\sigma_{-1}: \mathfrak{g}_{-1} \rightarrow \mathfrak{g}_{0}$ are $\mathbf{R}$-linear mappings such that

$$
\begin{gathered}
\sigma_{-2}\left(\left[X_{-1}, Y_{-1}\right]\right)=\sigma_{-1}\left(X_{-1}\right)\left(Y_{-1}\right)-\sigma_{-1}\left(Y_{-1}\right)\left(X_{-1}\right), \\
0=\sigma_{-1}\left(X_{-1}\right)\left(X_{-2}\right)+\left[X_{-1}, \sigma_{-2}\left(X_{-2}\right)\right]
\end{gathered}
$$

for all $X_{-1}, Y_{-1} \in \mathrm{g}_{-1}$ and $X_{-2} \in \mathrm{g}_{-2}$.

By Proposition 3.2, we can write $\sigma_{-2}\left(e_{\alpha}\right)=\zeta_{\alpha}^{k} e_{k}$ and $\sigma_{-1}\left(e_{i}\right)=\left(a_{i}, \tau_{i}\right), \sigma_{-1}\left(J e_{i}\right)=$ $\left(a_{i}^{\prime}, \tau_{i}^{\prime}\right)$, where $\zeta_{\alpha}^{k} \in \mathbf{C}, a_{i}, a_{i}^{\prime} \in R$, and $\tau_{i}, \tau_{i}^{\prime}$ are diagonal matrices satisfying (3.12).

Put $X_{-1}=\xi^{i} e_{i}$ and $Y_{-1}=\xi^{\prime \prime} e_{j}$ in (3.15), where $\xi^{i}, \xi^{\prime j} \in \mathbf{R}$. We obtain, using (3.3),

$$
\sqrt{-1} g_{i j}^{\alpha}\left(\xi^{i} \xi^{j}-\xi^{i} \xi^{j}\right) \zeta_{\alpha}^{k} e_{k}=\xi^{i}\left(\frac{1}{2} a_{i}+\tau_{i}^{k}\right) \xi^{\prime k} e_{k}-\xi^{\prime \prime}\left(\frac{1}{2} a_{i}+\tau_{i}^{k}\right) \xi^{k} e_{k},
$$

where we have written $\tau_{i}=\operatorname{diag}\left(\tau_{i}^{1}, \ldots, \tau_{i}^{n}\right)$. Since $e_{k}$ are linearly independent over $\mathbf{C}$ and $\xi^{i}, \xi^{j}$ are arbitrary, we deduce

$$
\sqrt{-1}\left(g_{i j}^{\alpha}-g_{j i}^{\alpha}\right) \zeta_{\alpha}^{k}=\delta_{j}^{k}\left(\frac{1}{2} a_{i}+\tau_{i}^{k}\right)-\delta_{i}^{k}\left(\frac{1}{2} a_{j}+\tau_{j}^{k}\right) .
$$

Recall that $g_{i j}^{\alpha} \neq 0$ only if $j=\tilde{i}$. For given $i$, take $j$ different from $i$ and $\tilde{i}$ and $k=j$. Then

$$
\frac{1}{2} a_{i}+\tau_{i}^{j}=0 \text { for all } j \neq i, j \neq \tilde{i}
$$

Take the conjugate using (3.13), and deduce

$$
\frac{1}{2} a_{i}-\tau_{i}^{j}=0 \text { for all } j \neq i, j \neq \tilde{i} .
$$

Consequently $a_{i}=0$ (and $\tau_{i}^{j}=0$ for all $j$ different from $\left.i, i\right)$. Similarly $a_{i}^{\prime}=0$.

By the representation in Proposition 3.2, we therefore see that $\sigma_{-1}\left(X_{-1}\right)\left(X_{-2}\right)=0$ for all $X_{-1} \in g_{-1}$ and $X_{-2} \in \mathfrak{g}_{-2}$. Therefore (3.16), together with the nondegeneracy of [ , ], implies that $\sigma_{-2}=0$. Then (3.15) reduces to:

$$
\sigma_{-1}(X)(Y)=\sigma_{-1}(Y)(X) \text { for all } X, Y \in \mathfrak{g}_{-1} \text {. }
$$

Define $\langle X, Y\rangle=[X, J Y]$ for all $X, Y \in \mathrm{g}_{-1}$, so that $\langle$,$\rangle is a nondegenerate$ symmetric bilinear form on $\mathfrak{g}_{-1}$. For every $Z \in \mathfrak{g}_{-1}$, we have seen that $\sigma_{-1}(Z)$ is zero on $g_{-2}$, so from (3.4) we get

$$
\left[\sigma_{-1}(Z)(X), Y\right]+\left[\cdot X, \sigma_{-1}(Z)(Y)\right]=0,
$$

or

$$
\left\langle\sigma_{-1}(Z)(X), Y\right\rangle=-\left\langle X, \sigma_{-1}(Z)(Y)\right\rangle
$$

for all $X, Y, Z \in g_{-1}$, since $\sigma_{-1}(Z)$ is complex linear on $g_{-1}$. This, with (3.19) and the usual proof of the vanishing of the first prolongation of $o(n, R)$, shows that $\sigma_{-1}(Z)$ is zero on $g_{-1}$ for all $Z \in g_{-1}$. Summarizing, we have: 
THEOREM 3.4. Let $\mathrm{g}_{-2} \oplus \mathrm{g}_{-1}$ be a pseudo-complex graded Lie algebra with $\operatorname{dim}_{\mathbf{R}} \mathrm{g}_{-2}$ $=2, \operatorname{dim}_{\mathbf{C}} \mathfrak{g}_{-1}=n \geqslant 3$, defined by a most nondegenerate Hermitian map L: $\mathfrak{g}_{-1} \times$ $\mathrm{g}_{-1} \rightarrow \mathfrak{g}_{-2}$. Let $g^{\alpha}=\left(g_{i j}^{\alpha}\right)_{i, j}$ be matrices defined by (3.1) with respect to bases chosen according to Lemma 2.1. Then the prolongation of $\mathrm{g}_{-2} \oplus \mathrm{g}_{-1}$ is $\mathrm{g}_{-2} \oplus \mathrm{g}_{-1} \oplus \mathrm{g}_{0}$, and has a representation in $\operatorname{aff}(n+2, \mathrm{C}) \subset \mathrm{gl}(n+3, \mathrm{C})$ by means of block matrices, given by

$$
\left\{\left[\begin{array}{ll|l|l}
a & 0 & \zeta_{j}^{\left(1^{\prime}\right)} & \sqrt{-1} x \\
0 & a & \zeta_{j}^{\left(2^{\prime}\right)} & \sqrt{-1} y \\
\hline 0 & 0 & \frac{1}{2} a I+\tau & \zeta^{i} \\
\hline 0 & 0 & 0 & 0
\end{array}\right]: a, x, y \in \mathbf{R}, \zeta^{i} \in \mathbf{C}, \tau=\operatorname{diag}\left(\tau^{i}\right) \text { with } \tau^{i}=-\bar{\tau}^{i}\right\},
$$

where $\zeta_{j}^{(\alpha)}$ are defined by (3.2) and $\tilde{i}$ by (2.4).

4. The equivalence problem. Now that the finite dimensionality of the prolongation of the graded Lie algebra associated to a real submanifold of codimension 2 is established, we can proceed to solve the pseudoconformal equivalence problem.

Let $M$ be an RC-regular $(2 n+2)$-dimensional real submanifold of $\mathbf{C}^{n+2}(n>3)$, whose Levi form $L$ at every point is most nondegenerate. Let $\left\{e_{1}, \ldots, e_{n}\right.$, $\left.J e_{1}, \ldots, J e_{n}\right\}$ be a basis of $H(M)$ in a coordinate neighborhood, and $\left\{e_{1^{\prime}}, e_{2^{\prime}}\right\}$ a basis of $T(M) / H(M)$, such that $g^{\alpha}\left(\alpha=1^{\prime}, 2^{\prime}\right)$ are in the standard forms of Lemma 2.1. Fix three real parameters among $\lambda_{i}$, and the order of $\lambda_{i}$. Then by Lemma $2.2, e_{1^{\prime}}$ and $e_{2^{\prime}}$ are uniquely determined up to a common scalar multiple. They have representatives $\tilde{e}_{1^{\prime}}, \tilde{e}_{2^{\prime}} \in T(M)$ defined up to linear combinations of $e_{i}$, $J e_{j}$. Let $\left\{\theta^{1^{\prime}}, \theta^{2^{\prime}}, \theta_{1}^{i}, \theta_{2}^{j}\right\}$ be the dual basis of $\left\{\tilde{e}_{1^{\prime}}, \tilde{e}_{2^{\prime}}, e_{i}, J e_{j}\right\}$. Then $\theta^{\alpha}$ are uniquely determined by $e_{\alpha}$. We therefore have a well defined line bundle over $M$ consisting of all multiples $u \theta^{1^{\prime}}$ with $u \in \mathbf{R}$. Since we are interested in only the local problem, there is no loss of generality in taking the positive ray bundle $E$ consisting of all $u \theta^{1^{\prime}}$ with $u>0$. On $E$, the forms $\omega^{\alpha}$ which are the liftings of $u \theta^{\alpha}$ are well defined.

On $M$, define the complex forms $\theta^{i}=\theta_{1}^{i}+\sqrt{-1} \theta_{2}^{i}$ for $i=1, \ldots, n$. The dual of the relation

$$
L(X, Y) \equiv[X, J Y] \bmod X_{i}, J X_{j}
$$

is

$$
d \theta^{\alpha} \equiv-\sqrt{-1} g_{i j}^{\alpha} \theta^{i} \wedge \bar{\theta}^{j} \bmod \theta^{1^{\prime}}, \theta^{2^{\prime}}
$$

where $g_{i j}^{\alpha}$ are defined by (2.3). Since $g^{\alpha}$ are assumed to be in standard form, the only nonzero entries are $g_{i i}^{\alpha}$ with $\tilde{i}$ defined by (2.4). Write $g_{i}^{\alpha}=g_{i i}^{\alpha}$, so

$$
\begin{aligned}
d \theta^{\alpha}= & -\sqrt{-1} g_{i}^{\alpha} \theta^{i} \wedge \bar{\theta}^{i}+A_{\beta i}^{\alpha} \theta^{\beta} \wedge \theta^{i} \\
& +\bar{A}_{\beta i}^{\alpha} \theta^{\beta} \wedge \bar{\theta}^{i}+A^{\alpha} \theta^{1^{\prime}} \wedge \theta^{2^{\prime}}
\end{aligned}
$$

for each $\alpha$ (summation over $\beta, i)$. 
LemmA 4.1. Under a transformation $\theta^{* i}=\theta^{i}+B_{\gamma}^{i} \theta^{\gamma}$, the coefficients $B_{\gamma}^{i}$ are uniquely determined by $A_{2^{\prime} i}^{1^{\prime}}=A_{1^{\prime} i}^{2^{\prime}}=0$.

Proof. Let $A_{\beta i}^{* \alpha}, A^{* \alpha}$ be the new coefficients when $\theta^{i}$ are substituted by $\theta^{* i}$ in (4.2). Equating the coefficients of $\theta^{\beta} \wedge \theta^{i}$, we get

$$
A_{\beta i}^{\alpha}=\sqrt{-1} g_{i}^{\alpha} \bar{B}_{\beta}^{i}+A_{\beta i}^{* \alpha}
$$

for each $\alpha, \beta, i$. Since $g_{i}^{\alpha}$ are nonzero, the assertion follows.

Assuming that the transformation of Lemma 4.1 has been done, we can now write

$$
\begin{aligned}
d \theta^{\alpha}= & -\sqrt{-1} g_{i}^{\alpha} \theta^{i} \wedge \bar{\theta}^{i}+A_{i}^{\alpha} \theta^{\alpha} \wedge \theta^{i} \\
& +\bar{A}_{i}^{\alpha} \theta^{\alpha} \wedge \bar{\theta}^{i}+A^{\alpha} \theta^{1^{\prime}} \wedge \theta^{2^{\prime}}
\end{aligned}
$$

for each $\alpha$.

On $E, \omega^{\alpha}=u \theta^{\alpha}$, so $d \omega^{\alpha}=d u \wedge \theta^{\alpha}+u d \theta^{\alpha}$, and we obtain from (4.3),

$$
\begin{aligned}
d \omega^{\alpha}= & -\sqrt{-1} g_{i}^{\alpha} \psi^{i} \wedge \bar{\psi}^{i}+\omega^{\alpha} \wedge \phi+A_{i}^{\alpha} \omega^{\alpha} \wedge \psi^{i} \\
& +\bar{A}_{i}^{\alpha} \omega^{\alpha} \wedge \bar{\psi}^{i}+A^{\alpha} \omega^{1^{\prime}} \wedge \omega^{2^{\prime}},
\end{aligned}
$$

where $\psi^{i}=\sqrt{u} \theta^{i}, \phi=-d u / u$, and $A_{i}^{\alpha}$ and $A^{\alpha}$ are different from the previous values by functions of $u$. The dual of Lemma 2.1 then yields

Proposition 4.2. The collection of all $\left(\psi^{1}, \ldots, \psi^{n}\right)$ satisfying (4.4) forms a principal $G_{1}$-bundle $Y$ over $E$, where $G_{1}=\left\{\exp \tau: \tau=\operatorname{diag}\left(t^{i}\right), t^{i}=-\bar{t}^{i}\right\} . Y$ is $a$ principal $G_{0}$-bundle over $M$, where

$$
G_{0}=\left\{\left[\begin{array}{ll|l}
u & 0 & \\
0 & u & \\
\hline & & \sqrt{u} \exp \tau
\end{array}\right]: u>0, \tau=\operatorname{diag}\left(t^{i}\right), t^{i}=-\bar{t}^{i}\right\} .
$$

If we use $t^{i}$ as the fiber coordinates in $Y \rightarrow E$, then the forms $\omega^{i}=e^{i} \psi^{i}$ are well defined on $Y$, and (4.4) lifted to $Y$ reads

$$
d \omega^{\alpha}=-\sqrt{-1} g_{i}^{\alpha} \omega^{i} \wedge \bar{\omega}^{\tilde{i}}+\omega^{\alpha} \wedge \phi+A_{i}^{\alpha} \omega^{\alpha} \wedge \omega^{i}+\bar{A}_{i}^{\alpha} \omega^{\alpha} \wedge \bar{\omega}^{i}+A^{\alpha} \omega^{1^{\prime}} \wedge \omega^{2^{\prime}}
$$

where $A_{i}^{\alpha}$ differ from the previous values by functions of $t^{i}$. We note that $\omega^{\alpha}, \omega^{i}$, $\bar{\omega}^{j}, \phi$ are linearly independent, and $\phi$ is indeterminate by linear combinations of $\omega^{i}$, $\bar{\omega}^{j}$ and $\omega^{\gamma}$.

LEMMA 4.3. Under a transformation $\phi^{*}=\phi+B_{i} \omega^{i}+\bar{B}_{i} \bar{\omega}^{i}+B_{\gamma} \omega^{\gamma}$, the coefficients $B_{i}, B_{\gamma}$ are uniquely determined by $A_{i}^{1^{\prime}}+A_{i}^{2^{\prime}}=0$ and $A^{\alpha}=0$.

Proof. Let $A_{i}^{* \alpha}, A^{* \alpha}$ be the new coefficients when $\phi^{*}$ is substituted for $\phi$ in (4.6). Then

$$
A_{i}^{* \alpha}+B_{i}=A_{i}^{\alpha} \quad \text { for each } \alpha, i
$$


and

$$
A^{* \alpha} \omega^{1^{\prime}} \wedge \omega^{2^{\prime}}+B_{\gamma} \omega^{\alpha} \wedge \omega^{\gamma}=A^{\alpha} \text { for each } \alpha .
$$

The assertion is now clear.

Summarizing so far, we have uniquely determined linearly independent forms $\omega^{\alpha}, \omega^{i}, \bar{\omega}^{j}, \phi$ on $Y$ such that

$$
\begin{aligned}
& d \omega^{\alpha}=-\sqrt{-1} g_{i}^{\alpha} \omega^{i} \wedge \bar{\omega}^{i}+\omega^{\alpha} \wedge \phi+A_{i}^{\alpha} \omega^{\alpha} \wedge \omega^{i}+\bar{A}_{i}^{\alpha} \omega^{\alpha} \wedge \bar{\omega}^{i}, \\
& A_{i}^{1^{\prime}}+A_{i}^{2^{\prime}}=0 \text { for each } i \text {. }
\end{aligned}
$$

Before we look at $d \omega^{i}$, we first notice that, when $M$ is given by two real equations $r^{\alpha}\left(z^{\beta}, z^{i}\right)=0, \alpha=1^{\prime}, 2^{\prime}$, we can take $\theta^{\alpha}=i \partial r^{\alpha}$ and $\theta^{i}=d z^{i}$, so that for this choice of $\theta^{\alpha}, \theta^{i}$, we have $d \theta^{i}=0$. Therefore for any other choice of $\theta^{\alpha}, \theta^{i}$, the 2 -forms $d \theta^{i}$ lie in the differential ideal generated by $\theta^{\alpha}$ and $\theta^{i}$. We can therefore write

$$
d \omega^{i}=\omega^{j} \wedge \phi_{j}^{i}+\omega^{\beta} \wedge \psi_{\beta}^{i}
$$

for some 1-forms $\phi_{j}^{i}, \psi_{\beta}^{i}$.

LEMMA 4.4. $\phi_{j}^{i} \equiv 0 \bmod \omega^{k}, \bar{\omega}^{l}, \omega^{\gamma}$ if $i \neq j$, and $\phi_{i}^{i}=\frac{1}{2} \phi+\tau^{i}$ for some 1 -forms $\tau^{i}$ satisfying

$$
\tau^{i}+\bar{\tau}^{i} \equiv 0 \bmod \omega^{k}, \bar{\omega}^{l}, \omega^{\gamma}
$$

Proof. The exterior derivative of (4.7) is

$$
\begin{aligned}
0= & -\sqrt{-1} d g_{i}^{\alpha} \wedge \omega^{i} \wedge \bar{\omega}^{i}-\sqrt{-1} g_{i}^{\alpha} d \omega^{i} \wedge \bar{\omega}^{i}+\sqrt{-1} g_{i}^{\alpha} \omega^{i} \wedge d \bar{\omega}^{i} \\
& +d \omega^{\alpha} \wedge \phi-\omega^{\alpha} \wedge d \phi+d A_{i}^{\alpha} \wedge \omega^{\alpha} \wedge \omega^{i}+d \bar{A}_{i}^{\alpha} \wedge \omega^{\alpha} \wedge \bar{\omega}^{i} \\
& +d \omega^{\alpha} \wedge\left(A_{i}^{\alpha} \omega^{i}+\bar{A}_{i}^{\alpha} \bar{\omega}^{i}\right)-\omega^{\alpha} \wedge\left(A_{i}^{\alpha} d \omega^{i}+\bar{A}_{i}^{\alpha} d \bar{\omega}^{i}\right)
\end{aligned}
$$

for each $\alpha$. First disregard the terms involving $\omega^{\gamma}$ after substitution using (4.7) and (4.9), and get

$$
\begin{aligned}
0 \equiv & -\sqrt{-1} d g_{i}^{\alpha} \wedge \omega^{i} \wedge \bar{\omega}^{i}-\sqrt{-1} g_{i}^{\alpha} \omega^{j} \wedge \phi_{j}^{i} \wedge \bar{\omega}^{i} \\
& +\sqrt{-1} g_{i}^{\alpha} \omega^{i} \wedge \bar{\omega}^{j} \wedge \bar{\phi}_{j}^{i}-\sqrt{-1} g_{i}^{\alpha} \omega^{i} \wedge \bar{\omega}^{i} \wedge \phi \\
& -\sqrt{-1} g_{i}^{\alpha} \omega^{i} \wedge \bar{\omega}^{i} \wedge\left(A_{k}^{\alpha} \omega^{k}+\bar{A}_{k}^{\alpha} \bar{\omega}^{k}\right) \bmod \omega^{\gamma} .
\end{aligned}
$$

Note that $d g_{i}^{\alpha}$ involve only $\omega^{k}, \bar{\omega}^{l}$ and $\omega^{\gamma}$ since $g_{i}^{\alpha}$ are functions on $M$. Hence, modulo $\omega^{\gamma}$,

$$
\begin{aligned}
0 \equiv & \sqrt{-1} g_{j}^{\alpha} \omega^{i} \wedge \bar{\omega}^{j} \wedge \phi_{i}^{j}+\sqrt{-1} g_{i}^{\alpha} \omega^{i} \wedge \bar{\omega}^{j} \wedge \bar{\phi}_{j}^{i} \\
& -\sqrt{-1} g_{i}^{\alpha} \delta_{i j} \omega^{i} \wedge \bar{\omega}^{j} \wedge \phi+\left(\text { terms involving only } \omega^{k}, \bar{\omega}^{l}\right)
\end{aligned}
$$

where $\delta_{i j}$ is the Kronecker delta. Hence

$$
g_{j}^{\alpha} \phi_{i}^{\tilde{j}}+g_{i}^{\alpha} \bar{\phi}_{j}^{\tilde{i}}-g_{i}^{\alpha} \delta_{i j} \phi \equiv 0 \bmod \omega^{k}, \bar{\omega}^{l}, \omega^{\gamma} .
$$


If $i \neq \tilde{j}$,

$$
g_{j}^{\alpha} \phi_{i}^{j}+g_{i}^{\alpha} \bar{\phi}_{j}^{i} \equiv 0 \bmod \omega^{k}, \bar{\omega}^{l}, \omega^{\gamma} .
$$

Since $g_{i}^{1^{\prime}}$ are not proportional to $g_{i}^{2^{\prime}}$, this implies that $\phi_{i}^{j} \equiv 0 \bmod \omega^{k}, \bar{\omega}^{l}, \omega^{\gamma}$. On the other hand, if $i=\tilde{j},(4.13)$ reduces to

$$
g_{i}^{\alpha} \phi_{i}^{i}+g_{i}^{\alpha} \bar{\phi}_{i}^{\tilde{i}} \equiv g_{i}^{\alpha} \phi \bmod \omega^{k}, \bar{\omega}^{l}, \omega^{\gamma} \text {. }
$$

Write

$$
\tau^{i}=\phi_{i}^{i}-\frac{1}{2} \phi \text { for each } i
$$

Then (4.10) follows.

Proposition 4.5. The 1-forms $\omega^{k}, \bar{\omega}^{l}, \omega^{\gamma}, \phi, \tau^{i}$ are linearly independent on $Y$.

Proof. From (4.9) and the definition $\omega^{i}=e^{i} \psi^{i}$, we have $\tau^{i}=(\ldots) d t^{i}+\ldots$ with nonzero coefficient. Here $t^{i}$ are fiber coordinates of $Y \rightarrow E$.

LEMMA 4.6. $\psi_{\beta}^{i} \equiv 0 \bmod \omega^{k}, \bar{\omega}^{l}, \omega^{\gamma}$.

Proof. Fix $\alpha$ and look at the terms in (4.11) involving $\omega^{\beta}$ with $\beta \neq \alpha$. Using (4.7) and (4.9), we get

$$
\begin{aligned}
0= & -\sqrt{-1} g_{i}^{\alpha} \omega^{\beta} \wedge \psi_{\beta}^{i} \wedge \bar{\omega}^{i}+\sqrt{-1} g_{i}^{\alpha} \omega^{i} \wedge \omega^{\beta} \wedge \bar{\psi}_{\beta}^{i} \\
& +\omega^{\beta} \wedge\left(\text { terms involving only } \omega^{k}, \bar{\omega}^{l}\right)+\omega^{\alpha} \wedge \omega^{\beta} \wedge(\ldots) .
\end{aligned}
$$

The lemma easily follows.

From Lemmas 4.4 and 4.6, we can now write (4.9) as

$$
\begin{aligned}
d \omega^{i}= & \omega^{i} \wedge\left(\frac{1}{2} \phi+\tau^{i}\right)+B_{j k}^{i} \omega^{j} \wedge \omega^{k}+C_{k l}^{i} \omega^{k} \wedge \bar{\omega}^{l} \\
& +B_{\beta k}^{i} \omega^{\beta} \wedge \omega^{k}+C_{\beta l}^{i} \omega^{\beta} \wedge \bar{\omega}^{l}+D^{i} \omega^{1^{\prime}} \wedge \omega^{2^{\prime}},
\end{aligned}
$$

where $B_{j k}^{i}=-B_{k j}^{i}$ for all $i, j, k$.

LEMMA 4.7. Under a transformation

$$
\tau^{* i}=\tau^{i}+E_{k}^{i} \omega^{k}+F_{k}^{i} \bar{\omega}^{k}+E_{\beta}^{i} \omega^{\beta},
$$

the coefficients $E_{k}^{i}, F_{k}^{i}$ and $E_{\beta}^{i}$ are uniquely determined by the conditions

$$
\tau^{i}=-\bar{\tau}^{i}, \quad C_{i j}^{i}=0 \text { and } B_{\beta i}^{i}=\bar{B}_{\beta i}^{i} \quad \text { for all } i, j, \beta \text {. }
$$

Proof. By (4.10), we can write

$$
\tau^{i}+\bar{\tau}^{i}=G_{k}^{i} \omega^{k}+H_{k}^{i} \bar{\omega}^{k}+H_{\beta}^{i} \omega^{\beta}, \text { for each } i
$$

so

$$
\tau^{i}+\bar{\tau}^{\tilde{i}}=G_{k}^{\tilde{i}} \omega^{k}+H_{k}^{\tilde{i}} \bar{\omega}^{k}+H_{\beta}^{\tilde{i}} \omega^{\beta} .
$$

But the left-hand sides are conjugate, so

$$
G_{k}^{\tilde{i}}=\bar{H}_{k}^{i}, \quad H_{\beta}^{\tilde{i}}=\bar{H}_{\beta}^{i} .
$$


On the other hand, under the transformation (4.16), we have

$$
\begin{aligned}
\tau^{* i}+\bar{\tau}^{* i} & =\tau^{\tilde{i}}+\bar{\tau}^{i}+\left(E_{k}^{\tilde{i}} \omega^{k}+F_{k}^{\tilde{i}} \bar{\omega}^{k}+E_{\beta}^{\tilde{i}} \omega^{\beta}\right)+\left(\bar{E}_{k}^{i} \bar{\omega}^{k}+\bar{F}_{k}^{i} \omega^{k}+\bar{E}_{\beta}^{i} \omega^{\beta}\right) \\
& =\left(G_{k}^{i}+E_{k}^{i}+\bar{F}_{k}^{i}\right) \omega^{k}+\left(H_{k}^{i}+F_{k}^{i}+\bar{E}_{k}^{i}\right) \bar{\omega}^{k}+\left(H_{\beta}^{i}+E_{\beta}^{i}+\bar{E}_{\beta}^{i}\right) \omega^{\beta} .
\end{aligned}
$$

This can be set to equal zero by choosing, say,

$$
E_{k}^{\tilde{i}}=\bar{F}_{k}^{i}=-\frac{1}{2} G_{k}^{i}, \quad E_{\beta}^{\tilde{i}}=-\frac{1}{2} H_{\beta}^{i},
$$

because of (4.18). We can therefore assume that $\tau^{i}$ already satisfies $\tau^{i}=-\bar{\tau}^{i}$, and look at the transformation (4.16) where $\tau^{* i}=-\bar{\tau}^{* i}$, so

$$
\bar{F}_{k}^{i}+E_{k}^{\tilde{i}}=0, \quad \bar{E}_{\beta}^{i}+E_{\beta}^{\tilde{i}}=0 \text { for each } i, k, \beta .
$$

Let $\beta_{j k}^{* i}$, etc., be the coefficients in (4.15) when $\tau^{i}$ are replaced by $\tau^{* i}$. Then

$$
\begin{aligned}
\left(B_{j k}^{i}-B_{j k}^{* i}\right) \omega^{j} \wedge \omega^{k} & =E_{k}^{i} \omega^{i} \wedge \omega^{k}, \\
\left(C_{k l}^{i}-C_{k l}^{* i}\right) \omega^{k} \wedge \bar{\omega}^{l} & =F_{l}^{i} \omega^{i} \wedge \bar{\omega}^{l}, \\
\left(B_{\beta k}^{i}-B_{\beta k}^{* i}\right) \omega^{\beta} \wedge \omega^{k} & =-E_{\beta}^{i} \omega^{\beta} \wedge \omega^{i} .
\end{aligned}
$$

From (4.21), $C_{i l}^{i}-C_{i l}^{* i}=F_{l}^{i}$, so $F_{l}^{i}$, hence also $E_{l}^{i}$ by (4.19), are uniquely determined by $C_{i l}^{* i}=0$ for all $i, l$. From (4.22),

$$
B_{\beta i}^{* i}=B_{\beta i}^{i}+E_{\beta}^{i} \text { for all } i, \beta,
$$

so

$$
B_{\beta i}^{* i}=B_{\beta i}^{i}+E_{\beta}^{i} \text { for all } i, \beta .
$$

If we require $\bar{B}_{\beta i}^{* i}=B_{\beta \tilde{i}}^{* i}$ for all $i, \beta$, then $E_{\beta}^{i}$ are uniquely given by

$$
E_{\beta}^{i}=\frac{1}{2}\left(\bar{B}_{\beta i}^{i}-B_{\beta i}^{i}\right)
$$

because of (4.19). The proof of the lemma is now complete.

Summarizing, we have

THEOREM 4.8. Let $M$ be an RC-regular $(2 n+2)$-dimensional real submanifold of $\mathrm{C}^{n+2}$ with $n \geqslant 3$, whose Levi form $L$ at every point is most nondegenerate. Let $\mathrm{g}^{\alpha}$ $\left(\alpha=1^{\prime}, 2^{\prime}\right)$ be the matrices of the Levi form normalized according to Lemma 2.1. Fix three real parameters among the eigenvalues $\lambda_{i}$ of $\left(g^{1^{\prime}}\right)^{-1} g^{2^{\prime}}$, and the order of $\lambda_{i}$. Then locally there is a well defined $G_{0}$-bundle $Y$ over $M$, where $G_{0}$ is the group defined in (4.5), and uniquely defined forms $\omega^{i}, \bar{\omega}^{j}, \omega^{\alpha}, \phi, \tau^{i}$ which form a basis of the complexified cotangent bundle of $Y$, where $\omega^{\alpha}$ and $\phi$ are real, and $\tau^{i}=-\bar{\tau}^{i}$, satisfying the structure equations (4.7) and (4.15), subject to the conditions (4.8) and (4.17). Two such real analytic submanifolds are locally pseudo conformally equivalent if and only if there is a local diffeomorphism preserving the matrices $g^{\alpha}$ and the forms $\omega^{i}, \bar{\omega}^{j}, \omega^{\alpha}, \phi$, $\tau^{i}$ on the associated $G_{0}$-bundles.

Proof. Only the sufficiency of the last condition needs to be proved, and this can be done in the same way as [2, Theorem 4.6]. 
5. The case of equivalent Levi forms. We now assume that the Levi forms at all points are equivalent, so $g^{\alpha}$ are constant on $M$. By exploiting the identities $d d \omega^{\alpha}=0=d d \omega^{i}$, we will show that $d \phi$ and $d \tau^{i}$ do not involve $\phi$ and $\tau^{j}$. For any function $f$ on $Y$, let us write

$$
d f=f_{, i} \omega^{i}+f_{, i} \bar{\omega}^{i}+f_{, \alpha} \omega^{\alpha}+f_{, \phi} \phi+f_{,(i)} \tau^{i} .
$$

Proposition 5.1. $d \phi$ does not involve $\phi$ and $\tau^{j}$, and we have $A_{i, \phi}^{\alpha}=\frac{1}{2} A_{i}^{\alpha}$ and $A_{i,(j)}^{\alpha}=\delta_{i j} A_{i}^{\alpha}$ for each $\alpha, i, j$.

Proof. The exterior derivative of (4.7) yields (4.11). Substitute from (4.7) and (4.15) and look at the terms involving $\omega^{\gamma} \wedge \phi$ or $\omega^{\gamma} \wedge \tau^{i}$. Let $d^{\prime} \phi$ be the part of $d \phi$ involving $\phi$ and $\tau^{j}$. Then

$$
\begin{aligned}
0= & \left(A_{i}^{\alpha} \omega^{\alpha} \wedge \omega^{i}+\bar{A}_{i}^{\alpha} \omega^{\alpha} \wedge \bar{\omega}^{i}\right) \wedge \phi \\
& -\omega^{\alpha} \wedge d^{\prime} \phi+\left(A_{i, \phi}^{\alpha} \phi+A_{i,(j)}^{\alpha} \tau^{j}\right) \wedge \omega^{\alpha} \wedge \omega^{i} \\
& +\left(\bar{A}_{i, \phi}^{\alpha} \phi+\bar{A}_{i,(j)}^{\alpha} \tau^{j}\right) \wedge \omega^{\alpha} \wedge \bar{\omega}^{i} \\
& +\omega^{\alpha} \wedge \phi \wedge\left(A_{i}^{\alpha} \omega^{i}+\bar{A}_{i}^{\alpha} \bar{\omega}^{i}\right)-A_{i}^{\alpha} \omega^{\alpha} \wedge \omega^{i} \wedge\left(\frac{1}{2} \phi+\tau^{i}\right) \\
& -\bar{A}_{i}^{\alpha} \omega^{\alpha} \wedge \bar{\omega}^{i} \wedge\left(\frac{1}{2} \phi+\bar{\tau}^{i}\right) \\
= & \omega^{\alpha} \wedge\left[-d^{\prime} \phi+A_{i, \phi}^{\alpha} \omega^{i} \wedge \phi+A_{i,(j)}^{\alpha} \omega^{i} \wedge \tau^{j}+\bar{A}_{i, \phi}^{\alpha} \bar{\omega}^{i} \wedge \phi\right. \\
& \left.\quad+\bar{A}_{i,(j)}^{\alpha} \bar{\omega}^{i} \wedge \tau^{j}-A_{i}^{\alpha} \omega^{i} \wedge\left(\frac{1}{2} \phi+\tau^{i}\right)-\bar{A}_{i}^{\alpha} \bar{\omega}^{i} \wedge\left(\frac{1}{2} \phi+\bar{\tau}^{i}\right)\right] .
\end{aligned}
$$

Consequently

$$
\begin{aligned}
d^{\prime} \phi= & \left(A_{i, \phi}^{\alpha}-\frac{1}{2} A_{i}^{\alpha}\right) \omega^{i} \wedge \phi+\left(A_{i,(j)}^{\alpha}-A_{i}^{\alpha} \delta_{i j}\right) \omega^{i} \wedge \tau^{j} \\
& + \text { conjugates }+\omega^{\alpha} \wedge(\ldots) .
\end{aligned}
$$

This should hold for $\alpha=1^{\prime}, 2^{\prime}$, so the last term should be a multiple of $\omega^{1^{\prime}} \wedge \omega^{2^{\prime}}$, and can therefore be dropped.

Furthermore, we have

$$
A_{i, \phi}^{1^{\prime}}-\frac{1}{2} A_{i}^{1^{\prime}}=A_{i, \phi}^{2^{\prime}}-\frac{1}{2} A_{i}^{2^{\prime}} \text {. }
$$

Using (4.8), we therefore get $A_{i, \phi}^{\alpha}-\frac{1}{2} A_{i}^{\alpha}=0$, and similarly $A_{i,(j)}^{\alpha}-A_{i}^{\alpha} \delta_{i j}=0$. Hence $d^{\prime} \phi=0$.

Proposition 5.2. $B_{\beta i}^{i}=0$ for every $\beta, i$.

Proof. For fixed $\alpha$, the terms in (4.11) which involve $\omega^{\beta} \wedge \omega^{k} \wedge \bar{\omega}^{l}$ with $\beta \neq \alpha$ are

$$
0=-\sqrt{-1} g_{i}^{\alpha} B_{\beta k}^{i} \omega^{\beta} \wedge \omega^{k} \wedge \bar{\omega}^{i}+\sqrt{-1} g_{i}^{\alpha} \omega^{i} \wedge \bar{B}_{\beta l}^{i} \omega^{\beta} \wedge \bar{\omega}^{l},
$$

so $g_{i}^{\alpha} B_{\beta k}^{i}+g_{k}^{\alpha} \bar{B}_{\beta l}^{\tilde{k}}=0$ for each $k, l$ and for $\alpha \neq \beta$. Putting $k=\tilde{l}$ and using (4.17), we get $B_{\beta k}^{k}=0$ for each $\beta, k$.

Proposition 5.3. $B_{i k}^{i}$ is a constant multiple of $A_{k}^{\alpha}$ for each $i, k$, and $\alpha$. 
Proof. The terms involving $\omega^{j} \wedge \omega^{k} \wedge \bar{\omega}^{l}$ in (4.11) are

$$
\begin{aligned}
0= & -\sqrt{-1} g_{i}^{\alpha} B_{j k}^{i} \omega^{j} \wedge \omega^{k} \wedge \bar{\omega}^{i}+\sqrt{-1} g_{i}^{\alpha} \bar{C}_{k l}^{i} \omega^{i} \wedge \bar{\omega}^{k} \wedge \omega^{l} \\
& -\sqrt{-1} g_{i}^{\alpha} A_{k}^{\alpha} \omega^{i} \wedge \bar{\omega}^{i} \wedge \omega^{k} \\
= & -\sqrt{-1}\left[g_{i}^{\alpha} B_{j k}^{i}+g_{j}^{\alpha} \bar{C}_{l k}^{j}-g_{j}^{\alpha} A_{k}^{\alpha} \delta_{l j}\right] \omega^{j} \wedge \omega^{k} \wedge \bar{\omega}^{l} .
\end{aligned}
$$

Hence

$$
0=g_{i}^{\alpha} B_{j k}^{i}+g_{j}^{\alpha} \bar{C}_{l k}^{\tilde{j}}-g_{j}^{\alpha} A_{k}^{\alpha} \delta_{l j}-g_{i}^{\alpha} B_{k j}^{\tilde{l}}-g_{k}^{\alpha} \bar{C}_{l j}^{\tilde{k}}+g_{k}^{\alpha} A_{j}^{\alpha} \delta_{l \tilde{k}} .
$$

In particular, put $\tilde{l}=j \neq k$. Then, using (4.17), we obtain

$$
0=2 g_{j}^{\alpha} B_{j k}^{j}-g_{j}^{\alpha} A_{k}^{\alpha}-g_{k}^{\alpha} \bar{C}_{i j}^{\tilde{k}} \text { for } j \neq k, \text { for each } \alpha .
$$

Write this as

$$
g_{j}^{\alpha}\left(2 B_{j k}^{j}-A_{k}^{\alpha}\right)=g_{k}^{\alpha} \bar{C}_{\tilde{j}}^{\tilde{k}}
$$

Put $\alpha=1^{\prime}, 2^{\prime}$ and eliminate $\bar{C}_{\tilde{j}}^{\tilde{k}}$ to obtain

$$
g_{k}^{2^{\prime}} g_{j}^{1^{\prime}}\left(2 B_{j k}^{j}-A_{k}^{1^{\prime}}\right)=g_{k}^{1^{\prime}} g_{j}^{2^{\prime}}\left(2 B_{j k}^{j}-A_{k}^{2^{\prime}}\right),
$$

hence

$$
B_{j k}^{j}=A_{k}^{1^{\prime}}\left(g_{j}^{1^{\prime}} g_{k}^{2^{\prime}}+g_{k}^{1^{\prime}} g_{j}^{2^{\prime}}\right) / 2\left(g_{j}^{1^{\prime}} g_{k}^{2^{\prime}}-g_{k}^{1^{\prime}} g_{j}^{2^{\prime}}\right) \text {. }
$$

(Note that the denominator is nonzero by nonproportionality of $g_{j}^{1^{\prime}}$ and $g_{j}^{2^{\prime}}$.)

Proposition 5.4. $d \tau^{i}$ does not involve $\phi, \tau^{j}$.

Proof. The exterior derivative of (4.15) is

$$
\begin{aligned}
0= & d \omega^{i} \wedge\left(\frac{1}{2} \phi+\tau^{i}\right)-\omega^{i} \wedge\left(\frac{1}{2} d \phi+d \tau^{i}\right)+2 B_{j k}^{i} d \omega^{j} \wedge \omega^{k} \\
& +C_{k l}^{i} d \omega^{k} \wedge \bar{\omega}^{l}-C_{k l}^{i} \omega^{k} \wedge d \bar{\omega}^{l}+B_{\beta k}^{i}\left(d \omega^{\beta} \wedge \omega^{k}-\omega^{\beta} \wedge d \omega^{k}\right) \\
& +C_{\beta l}^{i}\left(d \omega^{\beta} \wedge \bar{\omega}^{l}-\omega^{\beta} \wedge d \bar{\omega}^{l}\right)+D^{i}\left(d \omega^{1^{\prime}} \wedge \omega^{2^{\prime}}-\omega^{1^{\prime}} \wedge d \omega^{2^{\prime}}\right) \\
& +d B_{j k}^{i} \wedge \omega^{j} \wedge \omega^{k}+d C_{k l}^{i} \wedge \omega^{k} \wedge \bar{\omega}^{l}+d B_{\beta k}^{i} \wedge \omega^{\beta} \wedge \omega^{k} \\
& +d C_{\beta l}^{i} \wedge \omega^{\beta} \wedge \bar{\omega}^{l}+d D^{i} \wedge \omega^{1^{\prime}} \wedge \omega^{2^{\prime}}
\end{aligned}
$$

Let $d^{\prime} \tau^{i}$ be the part of $d \tau^{i}$ involving $\phi$ or $\tau^{j}$. Substituting (4.7) and (4.15) in (5.3) and using (4.17) and Proposition 5.2 to simplify the terms involving $\omega^{i} \wedge \phi$ or $\omega^{i} \wedge \tau^{k}$, we obtain

$$
\begin{aligned}
0= & 2 B_{i k}^{i} \omega^{i} \wedge \omega^{k} \wedge\left(\frac{1}{2} \phi+\tau^{i}\right)-\omega^{i} \wedge d^{\prime} \tau^{i} \\
& +2 B_{i k}^{i} \omega^{i} \wedge\left(\frac{1}{2} \phi+\tau^{i}\right) \wedge \omega^{k}+2 B_{j i}^{i} \omega^{j} \wedge\left(\frac{1}{2} \phi+\tau^{j}\right) \wedge \omega^{i} \\
& +2\left(B_{i k, \phi}^{i} \phi+B_{i k,(j)}^{i} \tau^{j}\right) \wedge \omega^{i} \wedge \omega^{k} \\
= & \omega^{i} \wedge\left[-d^{\prime} \tau^{i}-2 B_{i k}^{i} \omega^{k} \wedge\left(\frac{1}{2} \phi+\tau^{k}\right)+2 \omega^{k} \wedge\left(B_{i k, \phi}^{i} \phi+B_{i k,(j)}^{i} \tau^{j}\right)\right] .
\end{aligned}
$$

Now from Propositions 5.1 and 5.3, we have $B_{i k, \phi}^{i}=\frac{1}{2} B_{i k}^{i}$ and $B_{i k,(j)}^{i}=\delta_{k j} B_{i k}^{i}$. Therefore $\omega^{i} \wedge d^{\prime} \tau^{i}=0$, which implies that 


$$
d^{\prime} \tau^{i}=\omega^{i} \wedge\left(K^{i} \phi+L_{j}^{i} \tau^{j}\right)
$$

for some functions $K^{i}$ and $L_{j}^{i}$. Then

$$
\begin{aligned}
d^{\prime} \tau^{i} & =\omega^{i} \wedge\left(K^{i} \phi+L_{j}^{i} \tau^{j}\right) \\
& =-d^{\prime} \bar{\tau}^{i}=-\bar{\omega}^{i} \wedge\left(\bar{K}^{i} \phi+\bar{L}_{j}^{i} \bar{\tau}^{j}\right) .
\end{aligned}
$$

Since $\omega^{i}$ occurs on one side but not the other, we conclude that $K^{i}=0=L_{j}^{i}$, so $d^{\prime} \tau^{i}=0$.

6. Simplification of the structure equations in special cases. The structure equations (4.7), (4.15) of a most nondegenerate real submanifold of codimension 2 involve quite a number of functions. In general these may not be identically zero (though explicit examples are hard to construct, owing to the need for diagonalizing matrices), making the formulas for $d \phi$ and $d \tau^{i}$ very complicated. We will therefore assume that some of these functions are identically zero, and derive some relations among the other functions.

First, assume that $g^{\alpha}$ are constant and $A_{i}^{\alpha}=0$ for each $\alpha, i$. Then equation (4.7) reduces to

$$
d \omega^{\alpha}=-\sqrt{-1} g_{i}^{\alpha} \omega^{i} \wedge \bar{\omega}^{i}+\omega^{\alpha} \wedge \phi .
$$

Its exterior derivative is

$$
0=-\sqrt{-1} g_{i}^{\alpha}\left(d \omega^{i} \wedge \bar{\omega}^{i}-\omega^{i} \wedge d \bar{\omega}^{i}\right)+d \omega^{\alpha} \wedge \phi-\omega^{\alpha} \wedge d \phi .
$$

Proposition 6.1. If $g^{\alpha}$ are constant and $A_{i}^{\alpha}=0$ for all $\alpha, i$ we have the following relations among $B_{\beta j}^{i}$ and $C_{\beta j}^{i}$ :

$$
\begin{aligned}
& g_{i}^{\alpha} B_{\beta j}^{i}=-g_{j}^{\alpha} \bar{B}_{\beta i}^{j} \text { for } \alpha \neq \beta, \\
& g_{j}^{1^{\prime} B_{1^{\prime} j}^{i}}=-g_{j}^{2^{\prime} B_{2^{\prime} j}^{i}}, \\
& g_{i}^{\alpha} C_{\beta j}^{i}=g_{j}^{\alpha} C_{\beta i}^{j} \text { for } \alpha \neq \beta, \\
& g_{j}^{1^{\prime} C^{i}{ }^{\prime} j}=-g_{j}^{2^{\prime} C_{2^{\prime} j}^{i}} \text { for } i \neq j .
\end{aligned}
$$

Furthermore,

$$
\begin{aligned}
d \phi= & -\sqrt{-1}\left(g_{i}^{\alpha} B_{\alpha k}^{i} \omega^{k} \wedge \bar{\omega}^{l}+g_{k}^{\alpha} \bar{B}_{\alpha l}^{\tilde{k}} \omega^{k} \wedge \bar{\omega}^{l}+g_{k}^{\alpha} \bar{C}_{\alpha l}^{\tilde{k}} \omega^{k} \wedge \omega^{l}+g_{i}^{\alpha} C_{\alpha k}^{i} \bar{\omega}^{k} \wedge \bar{\omega}^{l}\right) \\
& +\sqrt{-1}\left(g_{k}^{2^{\prime}} \omega^{1^{\prime}}-g_{k}^{1^{\prime}} \omega^{2^{\prime}}\right) \wedge\left(D^{k} \bar{\omega}^{\tilde{k}}-\bar{D}^{\tilde{k}} \omega^{k}\right) \\
& +E \omega^{1^{\prime}} \wedge \omega^{2^{\prime}}, \text { for each } \alpha .
\end{aligned}
$$

Proof. Substitute (4.15) and (6.1) in (6.2). The terms involving $\omega^{\gamma}$ are

$$
\begin{aligned}
0= & -\sqrt{-1} g_{i}^{\alpha}\left(B_{\beta k}^{i} \omega^{\beta} \wedge \omega^{k}+C_{\beta l}^{i} \omega^{\beta} \wedge \bar{\omega}^{l}+D^{i} \omega^{1^{\prime}} \wedge \omega^{2^{\prime}}\right) \wedge \bar{\omega}^{i} \\
& +\sqrt{-1} g_{i}^{\alpha} \omega^{i} \wedge\left(\bar{B}_{\beta l}^{i} \omega^{\beta} \wedge \bar{\omega}^{l}+\bar{C}_{\beta k}^{i} \omega^{\beta} \wedge \omega^{k}+\bar{D}^{i} \omega^{1^{\prime}} \wedge \omega^{2^{\prime}}\right) \\
& -\omega^{\alpha} \wedge d \phi .
\end{aligned}
$$


For a given $\alpha$, the coefficients of the terms $\omega^{\beta} \wedge \omega^{k} \wedge \bar{\omega}^{l}$ and $\omega^{\beta} \wedge \bar{\omega}^{k} \wedge \bar{\omega}^{l}$ with $\beta \neq \alpha$ give

$$
0=-\sqrt{-1}\left(g_{i}^{\alpha} B_{\beta k}^{\tilde{l}}+g_{k}^{\alpha} \bar{B}_{\beta l}^{\tilde{k}}\right), \quad 0=-\sqrt{-1}\left(g_{i}^{\alpha} C_{\beta k}^{\tilde{l}}-g_{\tilde{k}}^{\alpha} C_{\beta l}^{\tilde{k}}\right) .
$$

These are (6.3) and (6.5).

On the other hand, the terms involving $\omega^{\alpha}$ in (6.8) are

$$
\begin{aligned}
0= & \omega^{\alpha} \wedge\left[-\sqrt{-1} g_{i}^{\alpha}\left(B_{\alpha k}^{i} \omega^{k}+C_{\alpha l}^{i} \bar{\omega}^{l}\right) \wedge \bar{\omega}^{i}\right. \\
& \left.-\sqrt{-1} g_{i}^{\alpha} \omega^{i} \wedge\left(\bar{B}_{\alpha l}^{i} \bar{\omega}^{l}+\bar{C}_{\alpha k}^{i} \omega^{k}\right)-d \phi\right] \\
& +\sqrt{-1} g_{k}^{\alpha}\left(-D^{k} \bar{\omega}^{\tilde{k}}+\bar{D}^{\tilde{k}} \omega^{k}\right) \wedge \omega^{1^{\prime}} \wedge \omega^{2^{\prime}}
\end{aligned}
$$

Consequently

$$
\begin{aligned}
d \phi= & -\sqrt{-1} g_{l}^{\alpha} B_{\alpha k}^{i} \omega^{k} \wedge \bar{\omega}^{l}-\sqrt{-1} g_{k}^{\alpha} \bar{B}_{\alpha l}^{\bar{k}} \omega^{k} \wedge \bar{\omega}^{l} \\
& -\sqrt{-1} g_{i}^{\alpha} C_{\alpha k}^{i} \bar{\omega}^{k} \wedge \bar{\omega}^{l}-\sqrt{-1} g_{k}^{\alpha} \bar{C}_{\alpha l}^{\bar{k}} \omega^{k} \wedge \omega^{l} \\
& +\omega^{\gamma} \wedge \mu_{\gamma}, \text { for each } \alpha,
\end{aligned}
$$

for some 1-forms $\mu_{\gamma}$. Substitution back into (6.9) gives

$$
\mu_{1^{\prime}} \equiv \sqrt{-1} g_{k}^{2^{\prime}}\left(D^{k} \bar{\omega}^{k}-\bar{D}^{\tilde{k}} \omega^{k}\right) \bmod \omega^{\gamma}
$$

and

$$
\mu_{2^{\prime}} \equiv-\sqrt{-1} g_{k}^{1^{\prime}}\left(D^{k} \bar{\omega}^{\tilde{k}}-\bar{D}^{\tilde{k}} \omega^{k}\right) \bmod \omega^{\gamma},
$$

and hence (6.7). On the other hand, equating the expression (6.10) for $\alpha=1^{\prime}, 2^{\prime}$ gives

$$
\begin{aligned}
& g_{\tilde{l}}^{1^{\prime}} B_{1^{\prime} k}^{\tilde{i}}+g_{k}^{1^{\prime}} \bar{B}_{1^{\prime} l}^{\tilde{k}}=g_{i}^{2^{\prime}} B_{2^{\prime} k}^{\tilde{i}}+g_{k}^{2^{\prime}} \bar{B}_{2^{\prime} l}^{\tilde{k}}, \\
& g_{\tilde{l}}^{1^{\prime}} C_{1^{\prime} k}^{i}-g_{\tilde{k}}^{l^{\prime}} C_{1^{\prime} l}^{\tilde{k}}=g_{i}^{2^{\prime}} C_{2^{\prime} k}^{i}-g_{\tilde{k}}^{2^{\prime}} C_{2^{\prime} l}^{\tilde{k}} .
\end{aligned}
$$

Substitution of (6.3) into (6.11) to eliminate $\bar{B}_{\beta l}^{\tilde{k}}$ yields (6.4), making use of the nonproportionality of $g_{i}^{1^{\prime}}, g_{l}^{2^{\prime}}$ and Proposition 5.2. Similarly (6.5) and (6.12) yield (6.6).

For the purpose of the next proposition, we will write $\Delta_{i j}=g_{i}^{1^{\prime}} g_{j}^{2^{\prime}}-g_{i}^{2^{\prime}{ }^{1}{ }_{j}{ }^{\prime}}$ for each $i, j$, so $\Delta_{i j} \neq 0$ for $i \neq j$.

Proposition 6.2. If $g^{\alpha}$ are constant and $A_{i}^{\alpha}=0$ for all $\alpha, i$, we have

$$
\begin{aligned}
2 \Delta_{i k} B_{k j}^{i} & =-\Delta_{k j} \bar{C}_{l k}^{j}, \\
\Delta_{l j} C_{l k}^{j} & =\Delta_{l i} C_{l j}^{\tilde{k}}, \\
B_{k j}^{j} & =0=C_{k \tilde{k}}^{j},
\end{aligned}
$$

for all $j, k, l$. 
Proof. From (5.2), which holds under the assumption $g^{\alpha}=$ constant, putting $A_{i}^{\alpha}=0$ we get

$$
2 g_{i}^{\alpha} B_{k j}^{i}=g_{j}^{\alpha} \bar{C}_{l k}^{j}-g_{k}^{\alpha} \bar{C}_{l j}^{\bar{k}} \text { for all } j, k, l, \alpha .
$$

Putting $\alpha=1^{\prime}, 2^{\prime}$, we obtain two equations in $B_{k j}^{i}, \bar{C}_{l k}^{j}$ and $\bar{C}_{l j}^{\tilde{k}}$ from which we can eliminate one and obtain (6.13) and (6.14). Putting $\tilde{l}=j \neq k$ in (6.13) and using (4.17), we get $B_{k j}^{j}=0$ for $j \neq k$ (and hence for all $j, k$ ). Putting $\tilde{l}=k \neq j$ in (6.14) and using (4.17), we get $C_{\tilde{k} k}^{j}=0$ for $j \neq k$, and hence for all $j, k$.

COROLlary 6.3. Under the same assumptions, if $C_{j k}^{i}=0$ for all $i, j, k$, then $B_{j k}^{i}=0$ for all $i, j, k$.

Proof. For $i \neq j$, it follows from (6.13) by putting $\tilde{l} \neq k$; whereas for $i=j$ it follows from (6.15).

Next we assume further that $C_{j k}^{i}$ are identically zero.

THEOREM 6.4. Assume that $g^{\alpha}$ are constant and $A_{i}^{\alpha}=0=C_{j k}^{i}$ for all $\alpha, i, j, k$. Then all the functions appearing in (4.15) vanish, and the structure equations reduce to (6.1) and

$$
\begin{aligned}
d \omega^{i} & =\omega^{i} \wedge\left(\frac{1}{2} \phi+\tau^{i}\right), \\
d \phi & =0 \\
d \tau^{i} & =a^{i} \omega^{i} \wedge \bar{\omega}^{i}
\end{aligned}
$$

subject to $a^{i}=\bar{a}^{i}$.

Proof. Using Corollary 6.3, we rewrite (4.15) as

$$
\begin{aligned}
d \omega^{i}= & \omega^{i} \wedge\left(\frac{1}{2} \phi+\tau^{i}\right)+B_{\beta k}^{i} \omega^{\beta} \wedge \omega^{k}+C_{\beta l}^{i} \omega^{\beta} \wedge \bar{\omega}^{l} \\
& +D^{i} \omega^{1^{\prime}} \wedge \omega^{2^{\prime}}
\end{aligned}
$$

and its exterior derivative as

$$
\begin{aligned}
0= & d \omega^{i} \wedge\left(\frac{1}{2} \phi+\tau^{i}\right)-\omega^{i} \wedge\left(\frac{1}{2} d \phi+d \tau^{i}\right)+B_{\beta k}^{i}\left(d \omega^{\beta} \wedge \omega^{k}-\omega^{\beta} \wedge d \omega^{k}\right) \\
& +C_{\beta l}^{i}\left(d \omega^{\beta} \wedge \bar{\omega}^{l}-\omega^{\beta} \wedge d \bar{\omega}^{l}\right)+D^{i}\left(d \omega^{1^{\prime}} \wedge \omega^{2^{\prime}}-\omega^{1^{\prime}} \wedge d \omega^{2^{\prime}}\right) \\
& +d B_{\beta k}^{i} \wedge \omega^{\beta} \wedge \omega^{k}+d C_{\beta l}^{i} \wedge \omega^{\beta} \wedge \bar{\omega}^{l}+d D^{i} \wedge \omega^{1^{\prime}} \wedge \omega^{2^{\prime}}
\end{aligned}
$$

The terms in (6.20) involving $\omega^{i}$, after substituting from (6.1) and (6.19) and using $B_{\beta i}^{i}=0$, yield

$$
\begin{aligned}
& \frac{1}{2} d \phi+d \tau^{i} \equiv-\sqrt{-1} g_{i}^{\beta} B_{\beta k}^{i} \bar{\omega}^{i} \wedge \omega^{k}-B_{\beta k}^{i} B_{\gamma i}^{k} \omega^{\beta} \wedge \omega^{\gamma} \\
& -\sqrt{-1} g_{i}^{\beta} C_{\beta l}^{i} \bar{\omega}^{\tilde{i}} \wedge \bar{\omega}^{l}-C_{\beta k}^{i} \bar{C}_{\gamma i}^{k} \omega^{\beta} \wedge \omega^{\gamma} \\
& -\sqrt{-1} g_{i}^{1^{\prime}} D^{i} \bar{\omega}^{i} \wedge \omega^{2^{\prime}}+\sqrt{-1} g_{i}^{2^{\prime}} D^{i} \bar{\omega}^{i} \wedge \omega^{1^{\prime}} \\
& +B_{\beta k, i}^{i} \omega^{\beta} \wedge \omega^{k}+C_{\beta l, i}^{i} \omega^{\beta} \wedge \bar{\omega}^{l}+D_{, i}^{i} \omega^{1^{\prime}} \wedge \omega^{2^{\prime}},
\end{aligned}
$$


$\bmod \omega^{i}$ for each $i$. The conjugate of this equation with $i$ instead of $i$ gives a similar formula for $\frac{1}{2} d \phi+d \bar{\tau}^{\bar{i}}=\frac{1}{2} d \phi-d \tau^{i}$. The addition of these two formulas therefore gives

$$
\begin{aligned}
d \phi \equiv & -\left(B_{\beta k}^{i} B_{\gamma i}^{k}+C_{\beta k}^{i} \bar{C}_{\gamma i}^{k}+\bar{B}_{\beta k}^{i} \bar{B}_{\gamma \tilde{i}}^{k}+\bar{C}_{\beta k}^{i} C_{\gamma \tilde{i}}^{k}\right) \omega^{\beta} \wedge \omega^{\gamma} \\
& +B_{\beta k, i}^{i} \omega^{\beta} \wedge \omega^{k}+C_{\beta l, i}^{i} \omega^{\beta} \wedge \bar{\omega}^{l}+D_{, i}^{i} \omega^{1^{\prime}} \wedge \omega^{2^{\prime}} \\
& +\bar{B}_{\beta l, i}^{i} \omega^{\beta} \wedge \bar{\omega}^{l}+\bar{C}_{\beta k, i}^{i} \omega^{\beta} \wedge \omega^{k}+\bar{D}_{, i}^{i} \omega^{1^{\prime}} \wedge \omega^{2^{\prime}}
\end{aligned}
$$

$\bmod \omega^{i}$ and $\bar{\omega}^{i}$, for each $i$. Compare with (6.7). For any $k, l$, we can choose an index $i$ not equal to $k$ and $\tilde{l}$. Then the terms involving $\omega^{k} \wedge \bar{\omega}^{l}$ and $\omega^{k} \wedge \omega^{l}$ in (6.7) give

$$
g_{i}^{\alpha} B_{\alpha k}^{\tilde{l}}+g_{k}^{\alpha} \bar{B}_{\alpha l}^{\tilde{k}}=0=g_{k}^{\alpha} \bar{C}_{\alpha l}^{\tilde{k}}-g_{l}^{\alpha} \bar{C}_{\alpha k}^{\tilde{i}}
$$

for each $\alpha, k, l$. Combining with (6.3) and (6.5), using the nonproportionality of $g_{k}^{\mathbf{1}^{\prime}}$, $g_{k}^{2^{\prime}}$, we easily obtain $B_{\alpha l}^{k}=0=C_{\alpha l}^{\tilde{k}}$ for $k \neq l$ for all $\alpha$, thus $B_{\alpha l}^{k}=0$ for all $\alpha, k, l$. Now (6.21) reduces to

$$
d \phi \equiv\left(D_{, i}^{i}+\bar{D}_{, i}^{i}\right) \omega^{1^{\prime}} \wedge \omega^{2^{\prime}} \bmod \omega^{i} \text { and } \bar{\omega}^{i},
$$

for each $i$. For each $k$, we can choose $i \neq k$. Then the term in (6.7) involving $\omega^{1^{\prime}} \wedge \omega^{k}$ gives $D^{k}=0$, and then the term involving $\omega^{1^{\prime}} \wedge \omega^{2^{\prime}}$ gives $E=0$, and (6.17) is proved.

So far we have proved that all the functions appearing in (4.15) except $C_{\alpha i}^{i}$ vanish. For any $i$, the terms in (6.20) involving $\omega^{j} \wedge \omega^{k} \wedge \bar{\omega}^{l}$ now reduce to $0 \equiv-\sqrt{-1} g_{k}^{\beta} C_{\beta i}^{i} \omega^{k} \wedge \bar{\omega}^{\tilde{k}} \wedge \bar{\omega}^{i} \bmod \omega^{i}$, so for $k \neq i$ it follows that $C_{\alpha i}^{i}=0$, and (6.16) is proved. Finally, (6.18) follows easily from the exterior derivative of (6.16).

REMARK. In the proof of this theorem, the identities $d d \omega^{i}=0$ have not been fully exploited. It is conceivable that the theorem holds under weaker hypothesis, but other methods of attack seem necessary for its proof.

7. A standard pseudoconformally flat submanifold. In the complex projective space $\mathbf{C} \mathbf{P}^{n+2}$ there is a standard quartic of codimension 2 , denoted by $Q$, given in homogeneous coordinates $z^{1^{\prime}}, z^{2^{\prime}}, z^{1}, \ldots, z^{n}, z^{n+1}$ by the equations

$$
f^{\alpha} \equiv g_{j k}^{\alpha} z^{j} \bar{z}^{k}-z^{\alpha} \bar{z}^{n+1}-\bar{z}^{\alpha} z^{n+1}=0,
$$

$\alpha=1^{\prime}, 2^{\prime}$ (summation over $j, k=1, \ldots, n$ ) or in affine coordinates in $\mathbf{C}^{n+2}$, by

$$
g_{j k}^{\alpha} z^{j} \bar{z}^{k}-z^{\alpha}-\bar{z}^{\alpha}=0 .
$$

Here $g_{j k}^{\alpha}$ are given constants. We introduce the real forms $\theta^{1^{\prime}}, \theta^{2^{\prime}}$ and complex forms $\boldsymbol{\theta}^{1}, \ldots, \boldsymbol{\theta}^{n}$ on $Q \cap \mathbf{C}^{n+2}$ by

$$
\begin{aligned}
\theta^{\alpha} & =\sqrt{-1} \partial f^{\alpha} \quad \text { for } \alpha=1^{\prime}, 2^{\prime}, \\
\theta^{i} & =d z^{i} \quad \text { for } i=1, \ldots, n .
\end{aligned}
$$

These are linearly independent and satisfy

$$
d \theta^{\alpha}=-\sqrt{-1} g_{j k}^{\alpha} \theta^{j} \wedge \bar{\theta}^{k}
$$




$$
d \theta^{i}=0
$$

From these it follows easily that if $g^{\alpha}$ are both nonsingular and $\operatorname{det}\left(\lambda g^{1^{\prime}}-g^{2^{\prime}}\right)$ has all distinct roots, then $Q$ is a submanifold whose Levi form at every point is most nondegenerate; and if furthermore $n \geqslant 3$ and $g^{\alpha}$ are normalized as in Lemma 2.1 , the structure equations as constructed in $\$ 4$ are

$$
\begin{aligned}
d \omega^{\alpha} & =-\sqrt{-1} g_{j k}^{\alpha} \omega^{j} \wedge \bar{\omega}^{k}+\omega^{\alpha} \wedge \phi, \\
d \omega^{i} & =\omega^{i} \wedge\left(\frac{1}{2} \phi+\tau^{i}\right), \\
d \phi & =d \tau^{i}=0 .
\end{aligned}
$$

The submanifold $Q$ can therefore be called a pseudoconformally flat submanifold of $\mathbf{C P}^{n+2}$. It plays the same part as the usual real hyperquadric in the case of codimension one. Let $\bar{G}$ be the group of projective transformations of $\mathbf{C P}^{n+2}$ leaving $Q$ invariant. It is a quotient group (by the group $K$ of $(n+3)$ th roots of unity) of the subgroup $G^{\prime}$ of $\operatorname{SL}(n+3, C)$ leaving the Hermitian forms $f^{\alpha}$ of (7.1) invariant up to scalar multiples. Let $X^{\alpha}$ be the matrices of $f^{\alpha}$ with respect to the ordered basis $\left(z^{1^{\prime}}, z^{2^{\prime}}, z^{1}, \ldots, z^{n}, z^{n+1}\right)$ of $\mathbf{C}^{n+3}$. Then $G^{\prime}=\{g \in \operatorname{SL}(n+3, \mathrm{C})$ : ${ }^{t} g X^{\alpha} \bar{g}=r^{\alpha} X^{\alpha}$ for some $\left.r^{\alpha} \in R \backslash\{0\}\right\}$. Its Lie algebra $\bar{g}$ consists of the matrices $A \in \operatorname{sl}(n+3, \mathrm{C})$ such that ${ }^{t} A X^{\alpha}+X^{\alpha} \bar{A}=r^{\alpha} X^{\alpha}$ for some $r^{\alpha} \in R$. A routine calculation shows that $\overline{\mathrm{g}}$ is the same as the representation in $\operatorname{sl}(n+3, \mathrm{C})$ of the Lie algebra $g_{-2} \oplus g_{-1} \oplus g_{0}$ calculated in $\S 3$.

The group $\bar{G}$ is also isomorphic to a subgroup $G$ of

$$
\operatorname{Aff}(n+2, \mathrm{C}) \subset \mathrm{GL}(n+3, \mathrm{C})
$$

by means of the homomorphism $h: G^{\prime} \rightarrow \operatorname{Aff}(n+2, \mathrm{C})$ defined by $h(g)=b^{-1} g$, where $b$ is the $(n+3, n+3)$ th entry in the matrix $g$; $\operatorname{ker} h$ is the group $K$ of $(n+3)$ th roots of unity. The Lie algebra of $G$ is the same as $g=g_{-2} \oplus g_{-1} \oplus g_{0}$ of Theorem 3.4.

REMARK. The referee has pointed out that the material in this section is very close to work of S. Murakami (On automorphisms of Siegel domains, Lecture Notes in Math., vol. 286, Springer-Verlag, Berlin and New York, 1972).

8. The Cartan connection. Let $M$ be a $(2 n+2)$-dimensional submanifold of $\mathbf{C}^{n+2}$ $(n \geqslant 3)$, whose Levi forms at all points are equivalent and most nondegenerate, so $g_{j k}^{\alpha}$ are constants, which are assumed to be in standard form. Let $G$ be the group defined in the last section in terms of this Levi form. Let $G_{0}$ be the group defined by (4.5), considered as a subgroup of $G$. Recall that $Y$ is a $G_{0}$-principal bundle over $M$, and $\operatorname{dim} G=3 n+3=\operatorname{dim} G_{0}+\operatorname{dim} M$.

A Cartan connection [4] of type $G / G_{0}$ on the bundle $Y \rightarrow M$ is a g-valued 1-form $\omega$ on $Y$ such that (i) restricted to each fibre of $Y, \omega$ is the Maurer-Cartan form of $G_{0}$, (ii) $R_{g}^{*} \omega=\operatorname{Ad}\left(g^{-1}\right) \cdot \omega$ for all $g \in G_{0}$, (iii) $\omega(X) \neq 0$ for every nonzero tangent vector $X$ of $Y$. It can be considered as a connection in the associated $G$-principal bundle over $M$. 
Write the Maurer-Cartan form of $G$ as

$$
\left[\pi_{B}^{A}\right]=\left[\begin{array}{c|c|c}
\delta_{\beta}^{\alpha} \phi & g_{j k}^{\alpha} \bar{\omega}^{k} & \sqrt{-1} \omega^{\alpha} \\
\hline 0 & \delta_{j}^{i}\left(\frac{1}{2} \phi+\tau^{i}\right) & \omega^{i} \\
\hline 0 & 0 & 0
\end{array}\right],
$$

where $A, B$ run through $1^{\prime}, 2^{\prime}, 1, \ldots, n, n+1$, and $\alpha, \beta, i, j$ are as before, and there is summation over $k$. Here $\omega^{\alpha}, \phi$ are real forms and $\omega^{i}, \tau^{i}$ are complex forms satisfying $\tau^{i}=-\bar{\tau}^{i}$ for each $i$. Then the Maurer-Cartan equations $d \pi_{B}^{A}=-\pi_{C}^{A} \wedge \pi_{B}^{C}$ reduce to the equations (7.6). The Maurer-Cartan forms of $G_{0}$ are obtained by putting $\omega^{\alpha}$ and $\omega^{i}$ equal to zero.

Now given the submanifold $M$, we can construct the $G_{0}$-principal bundle $Y$ and the forms $\omega^{\alpha}, \omega^{i}, \phi, \tau^{i}$ as in $\S 4$, and define $\pi_{B}^{A}$ by (8.1). We then have a $g$-valued 1-form $\omega=\left(\pi_{B}^{A}\right)$ on $Y$ satisfying the conditions (1) and (3) for a Cartan connection. Let $\Pi_{B}^{A}=d \pi_{B}^{A}+\pi_{C}^{A} \wedge \pi_{B}^{C}$. Thus

$$
\begin{aligned}
\Pi_{n+1}^{\alpha}= & \sqrt{-1}\left(A_{k}^{\alpha} \omega^{\alpha} \wedge \omega^{k}+\bar{A}_{k}^{\alpha} \omega^{\alpha} \wedge \bar{\omega}^{k}\right), \\
\Pi_{n+1}^{i}= & B_{j k}^{i} \omega^{j} \wedge \omega^{k}+C_{k l}^{i} \omega^{k} \wedge \bar{\omega}^{l}+B_{\beta k}^{i} \omega^{\beta} \wedge \omega^{k} \\
& +C_{\beta l}^{i} \omega^{\beta} \wedge \bar{\omega}^{l}+D^{i} \omega^{1^{\prime}} \wedge \omega^{2^{\prime}} .
\end{aligned}
$$

Under the right translation by $g \in G_{0}$, it may be directly verified from the construction that $\omega^{\alpha}$ and $\omega^{i}$ transform according to $\operatorname{Ad}\left(g^{-1}\right)$. On the other hand, the forms $\phi$ and $\tau^{i}$ satisfying (4.7) and (4.15) are uniquely determined by the relations (4.8) and (4.17), which are invariant under the transformations

$$
\left(\pi_{B}^{A}\right) \rightarrow \operatorname{Ad}\left(g^{-1}\right) \cdot\left(\pi_{B}^{A}\right) \text { and }\left(\Pi_{B}^{A}\right) \rightarrow \operatorname{Ad}\left(g^{-1}\right) \cdot\left(\Pi_{B}^{A}\right) .
$$

Therefore we must have $R_{g}^{*} \omega=\left(\operatorname{Ad}\left(g^{-1}\right) \cdot \omega\right)$, and the theorem:

THEOREM 8.1. Let $M$ be a $(2 n+2)$-dimensional submanifold of $\mathbf{C}^{n+2}(n \geqslant 3)$ such that the Levi forms at all points are equivalent and most nondegenerate. Let $Y$ be the $G_{0}$-principal bundle over $M$ defined in $\$ 4$. Then there is a unique Cartan connection of type $G / G_{0}$ on the bundle $Y$ which is characterized by (4.8) and (4.17).

We call the unique Cartan connection in the above theorem the normal pseudoconformal connection of $M$.

Combining Theorems 4.8 and 8.1 , we see that the local pseudoconformal invariants of $M$ are all determined by the normal pseudoconformal connection.

\section{REFERENCES}

1. D. Burns, Jr. and S. Shnider, Pseudoconformal geometry of hypersurfaces in $\mathbf{C}^{n+1}$, Proc. Nat. Acad. Sci. U.S.A. 72 (1975), 2433-2436.

2. S. S. Chern and J. Moser, Real hypersurfaces in complex manifolds, Acta Math. 133 (1974), 219-271. MR 54 \# 13112.

3. R. Hermann, Convexity and pseudoconvexity for complex manifolds, J. Math. Mech. 13 (1964), 667-672. MR 29 \#5260.

4. S. Kobayashi, On connections of Cartan, Canad. J. Math. 8 (1956), 145-156. MR 17, 1126.

5. H. F. Lai, Characteristic classes of real manifolds immersed in complex manifolds, Trans. Amer. Math. Soc. 172 (1972), 1-33. MR 47 \#2618. 
6. N. Tanaka, On the pseudo-conformal geometry of hypersurfaces of the space of $n$ complex variables, $\mathrm{J}$. Math. Soc. Japan 14 (1962), 397-429. MR 26 \#3086.

7. , On generalized graded Lie algebras and geometric structures. I, J. Math. Soc. Japan 19 (1967), 215-254. MR 36 \#4470.

8. On differential systems, graded Lie algebras and pseudo-groups, J. Math. Kyoto Univ. 10 (1970), 1-82. MR 42 \#1165.

Department of Mathematics, Fordham University, BronX, New YorK 10458

Current address: Financial Strategies Group, Goldman, Sachs \& Company, 55 Broad Street, New York, New York 10004 